\title{
Random walks on the mapping class group
}

\author{
Joseph Maher*
}

October 29, 2018

\begin{abstract}
We show that a random walk on the mapping class group of an orientable surface gives rise to a pseudo-Anosov element with asymptotic probability one. Our methods apply to many subgroups of the mapping class group, including the Torelli group.

Keywords: random walk, mapping class group, complex of curves, pseudo-Anosov, Torelli group

Subject code: 37E30, 20H10, 60G50, 20F65.
\end{abstract}

\section{Contents}

1 Introduction 1

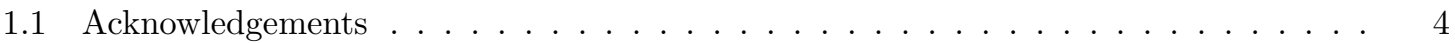

2 Preliminary definitions 4

2.1 Subgroups of the mapping class group . . . . . . . . . . . . . 8

3 Relative conjugacy bounds 11

3.1 Pseudo-Anosov elements . . . . . . . . . . . . . . . . . . . . . . . . 12

3.2 Reducible elements . . . . . . . . . . . . . . . . . . . . . . . . 14

3.3 Periodic elements . . . . . . . . . . . . . . . . . . . . . . . 16

4 Conjugates of relatively short elements 18

5 Random walks 23

5.1 Centralizers have harmonic measure zero . . . . . . . . . . . . . . 26

5.2 Asymptotic probabilities . . . . . . . . . . . . . . . . . 27

\section{Introduction}

Let $\Sigma$ be an orientable surface of finite type, i.e. a surface of genus $g$ with $p$ marked points, usually referred to as punctures. The mapping class group of $\Sigma$ consists of orientation preserving diffeomorphisms which preserve the punctures, modulo those isotopic to the identity. Thurston

\footnotetext{
*email: joseph.maher@csi.cuny.edu
} 
Thu88 showed that all elements of the mapping class group are periodic, reducible or pseudoAnosov. In this paper we show that a random walk on the mapping class group gives rise to a pseudo-Anosov element with asymptotic probability one. In fact we obtain a more general result which we now describe.

Let $\mu$ be a probability distribution on the mapping class group $G$. A random walk on $G$ is a Markov chain on $G$ with transition probabilities given by left translation of $\mu$, i.e. the probability that you go from $x$ at time $n$, to $y$ at time $n+1$, is $p(x, y)=\mu\left(x^{-1} y\right)$, and we shall assume that we start at the identity at time zero. The path space for the random walk is the probability space $\left(G^{\mathbb{Z}_{+}}, \mathbb{P}\right)$, where the product $G^{\mathbb{Z}_{+}}$is the collection of all sample paths, and the measure $\mathbb{P}$ is determined by $\mu$. If $w$ is a sample path, then we will write $w_{n}$ for the location of the sample path at time $n$, and the distribution of $w_{n}$ is given by the $n$-fold convolution of $\mu$. The support of the random walk is the semi-group generated by the support of $\mu$. We shall always assume that the group generated by the support of $\mu$ is a non-elementary subgroup of the mapping class group, i.e. it contains a pair of pseudo-Anosov elements with distinct fixed points in $\mathcal{P} \mathcal{M L}$. The mapping class group is finitely generated, so a choice of (finite) generating set gives rise to a word metric on the group, where the length of an element is the shortest length of any word in the generators equal to the group element, and two different choices of finite generating sets give quasi-isometric word metrics. We may also consider the word metric on the mapping class group with respect to an infinite generating set, though in this case the resulting metric need not be proper, and is often referred to as a relative metric. Masur and Minsky MM99 have shown that there is a choice of infinite generating set, consisting of a finite generating set union a particular collection of subgroups, which gives rise to a metric which is $\delta$-hyperbolic. In this case, we say that the mapping class group is weakly relatively hyperbolic. In fact, the mapping class group with this particular relative metric is quasi-isometric to the complex of curves. This gives two different ways to measure lengths of elements of the mapping class group, as we can measure their length using word length with respect to a finite generating set, or we can measure their length in the relative metric. The relative length of a group element is its distance from the identity in the relative metric. We show that $w_{n}$ is conjugate to an element of bounded relative length with asymptotic probability zero, i.e. the probability that $w_{n}$ is conjugate to an element of bounded relative length tends to zero as $n$ tends to infinity.

Theorem 1.1. Consider a random walk on the mapping class group of an orientable surface of finite type, which is not a sphere with three or fewer punctures, and let $w_{n}$ be the location of the random walk after $n$ steps. If the group generated by the support of the random walk is non-elementary, then for any constant $B, w_{n}$ is conjugate to an element of relative length at most $B$ with asymptotic probability zero.

There is a constant $B$, depending on the surface, such that any mapping class group element which is not pseudo-Anosov is conjugate to an element of relative length at most $B$, as shown in Lemma 5.5. and so this implies that $w_{n}$ is pseudo-Anosov with asymptotic probability one. Rivin Riv08 and Kowalski Kow08 have also shown that the nearest neighbour random walk on the mapping class group gives rise to a pseudo-Anosov element with asymptotic probability one, as part of a broader investigation of random walks on groups. Their methods apply whenever the support of the random walk maps onto $S p(2 n, \mathbb{Z})$, and have the advantage that they may be used to construct explicit lower bounds for the proportion of elements which are pseudo-Anosov at time $n$, and furthermore, show that the set of pseudo-Anosov elements is transient for these random walks. However, our methods apply to random walks supported on more general subgroups of the mapping class group. For example, the Torelli group is the subgroup of the mapping class group 
which acts trivially on the homology of the surface. This is a normal subgroup whose limit set is dense in the Thurston boundary, and so is not contained in a non-trivial centralizer. Therefore the nearest neighbour random walk on a Cayley graph for the Torelli group gives rise to a pseudo-Anosov element with asymptotic probability one.

We now give an outline of the main argument. Let $R$ be a set of elements in the mapping class group which are all conjugate to elements of bounded relative length, for example all of the nonpseudo-Anosov elements of the mapping class group. The basic idea is to consider the distribution of elements of $R$ inside the mapping class group, and show that random walks end up travelling through regions in which the density of elements of $R$ tends to zero. The long time behaviour of random walks in the mapping class group is described by harmonic measure on $\mathcal{P} \mathcal{M L}$, the space of projective measured laminations. This space may be thought of as a boundary for the mapping class group, following work of Masur and Minsky MM99 and Klarreich Kla, and we make this precise in Section 5. By work of Kaimanovich and Masur KM96, sample paths converge to the boundary with probability one, and this gives rise to a measure on $\mathcal{P} \mathcal{M L}$, called harmonic measure. The measure of a subset of the boundary is the probability that a sample path will converge to a lamination lying in that set, and this measure depends on the choice of probability distribution $\mu$ used to define the random walk. Given a subset $X$ of the mapping class group, we can take the harmonic measure of its limit set $\bar{X}$ in $\mathcal{P} \mathcal{M L}$. A sample path of the random walk is recurrent on $X$ if it hits $X$ infinitely often. If a sample path is recurrent on $X$, and it converges to the boundary, then it converges to a point in $\bar{X}$. As sample paths converge to the boundary with probability one, this means that the probability that a sample path is recurrent on $X$ is a lower bound on the harmonic measure of $\bar{X}$. In particular, if the harmonic measure of the limit set is zero, then the random walk is transient on $X$, i.e. a sample path hits $X$ only finitely many times with probability one.

In general the limit set of $R$ need not have harmonic measure zero, for example the set of all elements of the mapping class group which are not pseudo-Anosov has a limit set consisting of all of $\mathcal{P} \mathcal{M L}$, and so has harmonic measure one. However, we can consider $R_{k}$, the set of all the elements in $R$ which are word length distance (not relative distance) at most $k$ from some other element of $R$. We show that the limit set of $R_{k}$ is contained in the union of the limit sets of the centralizers of elements of the mapping class group of length at most $k$. We then show that this set has harmonic measure zero, assuming that the group $H$ generated by the support of the random walk is nonelementary, and every non-trivial element has a centralizer which has infinitely many images under $H$. Each element of $R \backslash R_{k}$ lives inside a ball of elements not in $R$ of radius at least $k$, and so there is an upper bound for the probability that a sample path hits an element of $R \backslash R_{k}$. This bound tends to zero as $k$ tends to infinity, so the probability that a sample path is not pseudo-Anosov tends to zero as the length of the path tends to infinity. Finally, if there are centralizers with finitely many images under the group $H$ generated by the support of the random walk, we show that we can map $H$ to the mapping class group of a surface covered by $\Sigma$, whose mapping class group does satisfy the condition on images of centralizers.

An important part of showing that the limit set of $R_{k}$ is a finite union of centralizers, is showing that if an element $g$ is conjugate to a relatively short element $s$, i.e. $g=w s w^{-1}$, and if $w$ is chosen to be the shortest conjugating word, then the path $w s w^{-1}$ is actually quasi-geodesic, for quasi-geodesic constants that depend on the relative length of $s$, but not of $g$. This follows from showing that the mapping class group has relative conjugacy bounds, which means that the relative length of a shortest conjugating word is bounded in terms of the relative lengths of the two conjugate words. Using further work of Masur and Minsky [MM00, we show that the mapping class group has relative conjugacy bounds by considering the action of the mapping class group on the complex of curves. 
The relative conjugacy bound property is equivalent to discreteness for the action of the mapping class group on the complex of curves, in the following sense: hyperbolic isometries have a minimal translation distance, and elliptic isometries have centralizers which act coarsely transitively on their coarse fixed sets.

The paper is structured as follows. In Section 2 we review some well known definitions and results. In Section 3 we show that the mapping class group has relative conjugacy bounds. In Section 4 we describe the distribution of $k$-dense reducible elements in the mapping class group, and finally in Section 5 we prove the main result on random walks.

\subsection{Acknowledgements}

I would like to thank Nathan Dunfield and Howard Masur for useful advice. I would especially like to thank the referees for pointing out many errors in an earlier version, supplying a correct proof of Lemma 5.8, and for suggestions on how to re-organize the paper. I would also like to thank Danny Calegari, Daniel Groves and Jason Manning for helpful conversations. Part of this paper was written while I was at the California Institute of Technology, and part while I was supported by a Postdoctoral Fellowship from the Centre de recherches mathématiques and the Institut des sciences mathématiques in Montréal. This work was also partially supported by NSF grant DMS-070674.

\section{Preliminary definitions}

In this section we review some of the definitions and results we will use in the main part of the paper, and fix some notation.

Let $\Sigma$ be the closed orientable surface of genus $g$ with $n$ marked points, also known as punctures. We will write $\Sigma_{g, n}$ if we need to explicit refer to the genus $g$, and the number of punctures $n$. The mapping class group of $\Sigma$ is the group of orientation preserving diffeomorphisms of the surface which preserve the set of punctures, modulo those isotopic to the identity. We say a surface is sporadic if it is a sphere with at most four punctures, or a torus with at most one puncture. If $\Sigma$ is a sphere with three or fewer punctures, then the mapping class group of $\Sigma$ is finite, and does not contain any pseudo-Anosov elements. If $\Sigma$ is a torus with at most one puncture, or a four punctured sphere, then the mapping class group is commensurable with $S L(2, \mathbb{Z})$, and random walks on $S L(2, \mathbb{Z})$ are well understood by work of Furstenberg [Fur71]. So for the remainder of this paper we will assume that the surface $\Sigma$ is not sporadic. However, we will at times need to consider subsurfaces of $\Sigma$ which may be sporadic, so certain results will be needed for surfaces which include a torus with one puncture, or a four punctured sphere.

Dehn Deh38 and Lickorish Lic64 showed that the mapping class group is finitely generated. In fact, we can describe an explicit generating set using Dehn twists. A Dehn twist is a map from the surface to itself defined by cutting the surface along an essential simple closed curve, and then gluing the two boundary components back together using a full twist. We say a simple closed curve in the surface is essential if it does not bound a subsurface which is either a disc, or a disc containing a single puncture. A Dehn twist represents a non-trivial element of the mapping class group. A Dehn twist is supported in a neighbourhood of the simple closed curve used to define it, and takes a transverse arc in a regular neighbourhood of the simple closed curve to one which winds once around the annular regular neighbourhood. This is illustrated in Figure 1 below. 

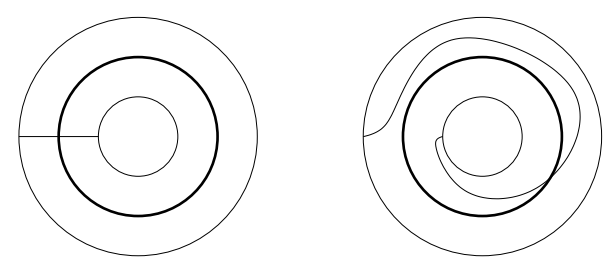

Figure 1: A Dehn twists in a simple closed curve.

The collection of Dehn twists in the simple closed curves shown below in Figure 2 generate the mapping class group of a closed surface with no punctures. If the surface has punctures, then there is a finite collection of Dehn twists which generate the finite index subgroup of the mapping class group which fixes each puncture, and this may be extended to a finite generating set for the whole group by adding elements which permute the punctures. These elements may be chosen to be half Dehn twist in simple closed curves that bound discs containing exactly two punctures, see for example Birman [Bir74, Chapter 4].

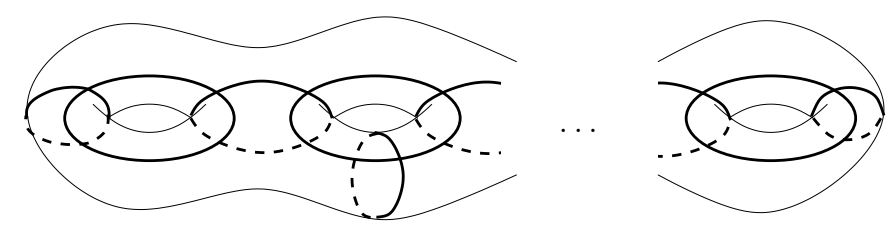

Figure 2: Dehn twists which generate the mapping class group.

We shall think of elements of the mapping class group as functions on surfaces, so we will write $a b$ to denote $a b(x)=a(b(x))$ for all $x \in \Sigma$.

The collection of essential simple closed curves in the surface may be made in to a simplicial complex, called the the complex of curves, which we shall denote $\mathcal{C}(\Sigma)$. The vertices of this complex are isotopy classes of simple closed curves in $\Sigma$, and a collection of vertices spans a simplex if representatives of the curves can be realised disjointly in the surface. The complex of curves is a finite dimensional simplicial complex, but it is not locally finite. We will write $\mathcal{C}_{0}(\Sigma)$ to denote the vertices of the simplicial complex $\mathcal{C}(\Sigma)$, which is the set of isotopy classes of simple closed curves. We will write $d_{\mathcal{C}}(x, y)$ for the distance in the one-skeleton between two vertices $x$ and $y$ of the complex of curves. We will always consider the complex of curves to have a basepoint $x_{0}$, which we shall take to be one of the curves corresponding to a standard generating set for the mapping class group. The mapping class group acts by simplicial isometries on the complex of curves. For certain sporadic surfaces the definition above produces a collection of disconnected points, and so a slightly different definition is used. If the surface is a torus with at most one puncture, then two vertices are connected by an edge if the corresponding simple closed curves may be isotoped to intersect transversely exactly once. If the surfaces is a four punctured sphere, then two vertices are connected by an edge if the corresponding simple closed curves may be isotoped to intersect transversely in two points. In these cases, the resulting curve complex is isomorphic to the Farey graph.

A geodesic metric space is $\delta$-hyperbolic if every geodesic triangle is $\delta$-slim, i.e. each edge is contained in a $\delta$-neighbourhood of the other two. Masur and Minsky MM99 have shown that the 
complex of curves is $\delta$-hyperbolic.

Theorem 2.1. MM99, Theorem 1.1] Let $\Sigma$ be an oriented surface of finite type. The curve complex $\mathcal{C}(\Sigma)$ is $\delta$-hyperbolic, and has infinite diameter unless $\Sigma$ is a sphere with three or fewer punctures.

An explicit bound for $\delta$ in terms of the complexity of the surface is given by Bowditch Bow06.

According to Thurston's classification of surface automorphisms, there are three sorts of elements of the mapping class group. We say that an element $g$ of the mapping class group is periodic if it has finite order. We say $g$ is reducible, if it fixes a disjoint collection of simple closed curves in $\Sigma$. The periodic and reducible elements act on the complex of curves as elliptic isometries. All other elements are pseudo-Anosov, and act on the complex of curves as hyperbolic isometries.

Let $G$ be a finitely generated group, together with a symmetric generating set $A$. For each element $g$ of $G$, we define the word length of $g$ to be the length of the shortest word representing $g$ in the generating set $A$. This length function on $G$ induces a left-invariant distance metric on $G$, called the word metric. This metric may also be obtained by forming the Cayley graph $\Gamma$ for the group. The Cayley graph is the graph whose vertices consist of elements of $G$, with two group elements $a$ and $b$ connected by an edge if $a^{-1} b$ is a member of the generating set. If we set the length of each edge equal to 1 then the distance between two vertices in the Cayley graph is the same as the word metric distance. Different choices of generating set give different, but quasi-isometric, word metrics. We will assume we have fixed some generating set for the mapping class group, and we will write $|x|$ for the word length of $x$, and $d(x, y)$ for the distance from $x$ to $y$ in the word metric on $G$. A geodesic in the word metric on $G$ is a word of minimal length in the generating set.

Given a group $G$, and a collection of subgroups $\mathcal{H}=\left\{H_{i}\right\}_{i \in I}$, we define the relative length of a group element $g$ to be the length of the shortest word in the typically infinite generating set $A \cup \mathcal{H}$. This defines a metric on $G$ called the relative metric, which depends on the choice of subgroups $\mathcal{H}$. We will write $\widehat{G}$ to denote the group $G$ with the relative metric, which we shall also refer to as the relative space. This space is quasi-isometric to the relative or electrified Cayley graph. The relative Cayley graph $\widehat{\Gamma}$ is the graph formed by adding a vertex for each coset $g H_{i}$, and then connecting this vertex to each element of the coset $g H_{i}$ by an edge of length one-half. However, we will find it convenient to work with $\widehat{G}$ rather than the electrified Cayley graph. We will write $|\widehat{x}|$ for the relative length of $x$, and $\widehat{d}(x, y)$ for the relative distance from $x$ to $y$. A geodesic in the relative space is a word of minimal length in the generators $A \cup \mathcal{H}$.

We say a finitely generated group $G$ is weakly relatively hyperbolic, relative to a finite list of subgroups $\mathcal{H}$, if the relative space $\widehat{G}$ is $\delta$-hyperbolic. This definition of relative hyperbolicity is due to Farb Far98, and is more general than the definition of strong relative hyperbolicity due to Gromov Gro87, see also Bowditch [Bow. Osin Osi06] contains a detailed discussion of several definitions of relative hyperbolicity, and their relations.

We may consider the relative metric on the mapping class group with respect to the following collection of subgroups. Let $\left\{\alpha_{1}, \ldots, \alpha_{n}\right\}$ be a list of representatives of orbits of simple closed curves in $\Sigma$, under the action of the mapping class group. We may choose the $\alpha_{i}$ to be distance at most one from the curves corresponding to the standard generators illustrated in Figure 2, Let $H_{i}=\operatorname{fix}\left(\alpha_{i}\right)$ be the subgroup of $G$ fixing $\alpha_{i}$. Masur and Minsky MM99 have shown that the resulting relative space is quasi-isometric to the complex of curves. As the complex of curves is $\delta$-hyperbolic, this shows that the mapping class group is weakly relatively hyperbolic.

Theorem 2.2. [MM99, Lemma 7.2] Let $\Gamma$ be a Cayley graph for the mapping class group, and let $\widehat{\Gamma}$ be the electrified Cayley graph with respect to subgroups corresponding to stabilizers of representatives of 
orbits of the mapping class group action on the vertices of the complex of curves. Then the electrified Cayley graph $\widehat{\Gamma}$ is quasi-isometric to the complex of curves $\mathcal{C}(\Sigma)$.

In fact, the mapping class group is not strongly relatively hyperbolic. There are a number of approaches to this available, of which Karlsson and Noskov KN04 seems to be the earliest, see also Bowditch [Bow05], Anderson, Aramayona and Shackleton [AAS07], and Behrstock, Drutu and Mosher BDM09] for a useful discussion of work in this area.

The quasi-isometry from the mapping class group with the relative metric to the complex of curves can be defined by sending a group element $g$ to $g\left(x_{0}\right)$, where $x_{0}$ is the basepoint of the complex of curves. We will always measure distances in $\mathcal{C}(\Sigma)$ between points that lie in the zeroskeleton, so the complex of curves is effectively a discrete space. Furthermore, the relative distance between two mapping class group elements is zero if and only if they are the same, so we only need an additive quasi-isometry constant on the right hand side of the inequalities below.

$$
\frac{1}{Q} \widehat{d}(g, h)-q \leqslant d_{\mathcal{C}}\left(g x_{0}, h x_{0}\right) \leqslant Q \widehat{d}(g, h) .
$$

The Gromov boundary of a $\delta$-hyperbolic space is the set of endpoints of quasi-geodesic rays, where two quasi-geodesic rays are said to be equivalent if they stay a bounded distance apart. The Gromov boundary of a non-locally compact space need not be compact, and in fact the Gromov boundary of the complex of curves is not compact. As the complex of curves and the relative space $\widehat{G}$ are quasi-isometric, we may identify their Gromov boundaries. If $X$ is a subset of either the complex of curves or the relative space, we will write $\bar{X}$ for the union of $X$ and its limit points in the Gromov boundary. The Gromov boundary of the complex of curves may be described in terms of Teichmüller space and the Thurston compactification of Teichmüller space.

Teichmüller space can be defined as the space of complete finite volume hyperbolic metrics on $\Sigma$, modulo isometries isotopic to the identity, which we shall denote $\mathcal{T}(\Sigma)$. Topologically this is homeomorphic to $\mathbb{R}^{6 g+2 p-6}$, where $g$ is the genus of $\Sigma$, and $p$ is the number of punctures. Thurston showed that the space of projective measured foliations $\mathcal{P} \mathcal{M F}$ can be used to compactify Teichmüller space in such a way that the action of the mapping class group extends continuously to $\mathcal{P} \mathcal{M F}$, which is a sphere of dimension $6 g+2 p-7$. The space of projective measured foliations $\mathcal{P} \mathcal{M F}$ is essentially the same as the space of projective measured laminations $\mathcal{P} \mathcal{M L}$, as the two spaces are homeomorphic by a homeomorphism which commutes with the action of the mapping class group.

Given $\epsilon>0$, for each $\gamma \in \mathcal{C}_{0}(\Sigma)$ define Thin $(\gamma)$ to be the subset of $\mathcal{T}(\Sigma)$ in which the length of the geodesic representative of $\gamma$ is at most $\epsilon$. We may choose $\epsilon$ to be sufficiently small so that some collection $\operatorname{Thin}\left(\gamma_{1}\right), \ldots, \operatorname{Thin}\left(\gamma_{n}\right)$ has non-empty intersection if and only if the curves $\gamma_{1}, \ldots, \gamma_{n}$ can be realised disjointly on the surface. Add a new point $x_{\gamma}$ for each simple closed curve $\gamma$, and a new edge of length one-half connecting $x_{\gamma}$ to each point in Thin $(\gamma)$. The resulting space is called electrified Teichmüller space, $\mathcal{T}_{e l}(\Sigma)$. Masur and Minsky MM99 show that $T_{e l}(\Sigma)$ is quasi-isometric to the complex of curves $\mathcal{C}(\Sigma)$, which is $\delta$-hyperbolic. In particular, this means their Gromov boundaries are the same.

Klarreich [Kla, Theorem 1.1], see also Hamenstädt [Ham06, has identified the Gromov boundary of the complex of curves with the space of minimal foliations on $\Sigma$, with the topology obtained from $\mathcal{P} \mathcal{M F}$ by forgetting the measures. We shall denote this space by $\mathcal{F}_{\text {min }}$, and the analogous space of laminations $\mathcal{L}_{\text {min }}$. A foliation is minimal if no leaf is a simple closed curve. Minimal foliations correspond to laminations which contain no simple closed curve, and which are not disjoint from any simple closed curves, and we will call such laminations filling laminations. A pseudo-Anosov 
element has a unique pair of fixed points in $\mathcal{P} \mathcal{M F}$ which are uniquely ergodic foliations, and in particular they are minimal foliations, and so a pseudo-Anosov element also has a unique pair of fixed points in $\mathcal{F}_{\text {min }}$ or $\mathcal{L}_{\text {min }}$.

\subsection{Subgroups of the mapping class group}

We now summarize various well-known properties of subgroups of the mapping class group that we will use in this paper. We provide arguments for the sake of completeness, but these are either elementary, or follow arguments from Ivanov Iva92.

Given a subgroup $F$ of $G$ we will write $C(F)$ for the centralizer of $F$, i.e. the subgroup of $G$ consisting of elements which commute with elements of $F$, and we shall write $N(F)$ for the normalizer of $F$, i.e. the subgroup of $G$ consisting of all elements $g$ such that $g F g^{-1}=F$. We will write $C(g)$ for the centralizer of the cyclic subgroup generated by $g$. If $F$ is finite, then the centralizer has finite index in the normalizer. A subgroup of the mapping class group is non-elementary if it contains a pair of pseudo-Anosov elements with distinct pairs of fixed points in the Thurston boundary $\mathcal{P} \mathcal{M L}$. A subgroup of the mapping class group is reducible if it preserves a finite collection of disjoint simple closed curves. Ivanov [Iva92 showed that any infinite subgroup of the mapping class group is either reducible or contains a pseudo-Anosov element, and the centralizer of a pseudo-Anosov element $g$ is virtually cyclic, and consists precisely of those elements which preserve the fixed points of $g$. Furthermore, if a subgroup consists entirely of periodic elements, then the subgroup is finite, see also Birman, Lubotzky and McCarthy, BLM83, McCarthy [McC85] and McCarthy and Papadopoulos MP89.

It will be useful to know certain properties of finite subgroups of the mapping class group. Let $F$ be a finite subgroup of the mapping class group. By the Nielsen realization theorem, due to Kerckhoff [Ker83, there is a hyperbolic structure for $\Sigma$ on which $F$ acts by isometries. Elements of $F$ need not act freely on $\Sigma$, so the quotient $\mathcal{O}=\Sigma / F$ may be an orbifold. Equivalently, in terms of conformal structures, there is an $F$-invariant conformal structure on $\Sigma$ and the quotient map $\Sigma \rightarrow \mathcal{O}$ is a branched cover. This covering is normal, and so gives rise to a map $\theta: \pi_{1} \mathcal{O} \rightarrow F$. The Teichmüller space of the quotient surface $\mathcal{T}(\mathcal{O})$ is the same as the Teichmüller space of the surface obtained by replacing all branch points with punctures, and is isometrically embedded in $\mathcal{T}(\Sigma)$ as the fixed point set of $F$, i.e. $\{x \in \mathcal{T}(\Sigma) \mid f(x)=x$ for all $f \in F\}$, which we shall write as $\operatorname{fix}_{\mathcal{T}}(F)$. The mapping class group of the orbifold $\mathcal{O}$, which we shall denote $G_{\mathcal{O}}$, consists of isotopy classes of homeomorphisms from $\mathcal{O}$ to itself, which preserve the set of punctures, and also preserve the sets of orbifold points or branch points of a given index.

We say a homeomorphism of $\Sigma$ is $F$-equivariant if it preserves pre-images of points under the covering map $\Sigma \rightarrow \mathcal{O}$. We will write $G_{F}$ for the subgroup of $G$ consisting of elements with $F$ equivariant representatives, which is also known as the relative mapping class group with respect to $F$. If an $F$-equivariant homeomorphism of $\Sigma$ is isotopic to the identity, then it is isotopic by an $F$-equivariant isotopy, see Maclachlan and Harvey [MH75, Zieschang Zie73] and Birman and Hilden [BH73], so this gives a well defined map $G_{F} \rightarrow G_{\mathcal{O}}$, with kernel $F$. We now show that the image of $G_{F}$ in $G_{\mathcal{O}}$ has finite index in $G_{\mathcal{O}}$.

Proposition 2.3. The image of the F-equivariant subgroup $G_{F}$ has finite index in the mapping class group $G_{\mathcal{O}}$ of the quotient surface $\mathcal{O}$.

Proof. A map $g: \mathcal{O} \rightarrow \mathcal{O}$ is covered by a map $\widetilde{g}: \Sigma \rightarrow \Sigma$ if and only if $g_{*}(\operatorname{ker} \theta) \subset \operatorname{ker} \theta$, where $\theta: \pi_{1} \mathcal{O} \rightarrow \pi_{1} \mathcal{O}$ is the map induced by the normal covering. As $F$ is finite, $\operatorname{ker} \theta$ is a finite index 
subgroup of $\pi_{1} \mathcal{O}$, and there are only finitely many finite index subgroups of that index, so the subgroup of $G_{\mathcal{O}}$ which leaves $\operatorname{ker} \theta$ invariant has finite index in $G_{\mathcal{O}}$. Therefore the image of $G_{F}$ has finite index in $G_{\mathcal{O}}$.

We define the complex of curves of an orbifold $\mathcal{C}(\mathcal{O})$ to be the complex of curves of the surface obtained by treating the orbifold points as punctures. The curve complex $C(\mathcal{O})$ is non-empty, unless $\mathcal{O}$ is a triangle orbifold. We will write $\operatorname{fix}_{K}(F)$ for the coarse fixed set of $F$ acting on the complex of curves $\mathcal{C}(\Sigma)$, i.e. all simple closed curves $x$ such that $d_{\mathcal{C}}(x, f x) \leqslant K$, for all $f \in F$. There is a map between the zero-skeletons $\mathcal{C}_{0}(\mathcal{O}) \rightarrow \mathcal{C}_{0}(\Sigma)$ which sends a simple closed curve in $\mathcal{O}$ to its pre-image in $\mathcal{C}_{0}(\Sigma)$, which is $F$-invariant and has diameter at most one. Therefore this map is coarsely well defined, and sends $\mathcal{C}_{0}(\mathcal{O})$ to fix $x_{1}(F)$, which is empty if $\mathcal{O}$ is a triangle orbifold. We say a group acts coarsely transitively on a metric space $(X, d)$ if there is a constant $K$ such that the $K$-neighbourhood of the orbit of a point in $X$ contains all of $X$. We now show that the centralizer $C(F)$ acts coarsely transitively on $\operatorname{fix}_{1}(F)$.

Proposition 2.4. Let $F$ be a finite subgroup of the mapping class group. Then the centralizer $C(F)$ acts coarsely transitively on fix $(F)$.

Proof. As $F$ is finite, the centralizer $C(F)$ has finite index in the normalizer $N(F)$. By Proposition 2.3. there is a homomorphism with finite kernel from the normalizer $N(F)$ onto a finite index subgroup of $G_{\mathcal{O}}$. The mapping class group $G_{\mathcal{O}}$ acts coarsely transitively on $\mathcal{C}(\mathcal{O})$, so $N(F)$ also acts coarsely transitively on $\mathcal{C}(\mathcal{O})$. The complex of curves $\mathcal{C}(\mathcal{O})$ maps coarsely onto $\operatorname{fix}_{1}(F)$, the set of all simple closed curves moved distance at most one by all elements of $F$, and so $N(F)$, and hence $C(F)$, acts coarsely transitively on $\operatorname{fix}_{1}(F)$.

In the case that $\mathcal{O}$ is a triangle orbifold, the mapping class group of $\mathcal{O}$, and hence the centralizer $C(F)$, is finite, and $\operatorname{fix}_{1}(F)$ is empty, so this statement is vacuously true.

The quasi-isometry from $\widehat{G}$ to $\mathcal{C}(\Sigma)$ is given by $g \mapsto g\left(x_{0}\right)$, where $x_{0}$ is a fixed basepoint in $\mathcal{C}(\Sigma)$. As $C(F)$ preserves fix $_{1}(F)$, the distance from the basepoint $x_{0}$ to fix $x_{1}(F)$ is the same as the distance from $g\left(x_{0}\right)$ to $\operatorname{fix}_{1}(F)$ for any $g \in N(F)$. This implies that the image of $N(F)$ lies in a bounded neighbourhood of fix $x_{1}(F)$, and by Proposition 2.4 the centralizer $C(F)$, and hence the normalizer $N(F)$, act coarsely transitively on $\operatorname{fix}_{1}(F)$, so they have the same limit sets in $\mathcal{L}_{\text {min }}$. We now observe that the limit set of $\operatorname{fix}_{1}(F)$, and hence the limit set of the centralizer $C(F)$, consists of the filling laminations fixed by $F$. We will write fix $\partial(F)$ for the fixed set of $F$ in the Gromov boundary $\partial \mathcal{C}(\Sigma)$.

Proposition 2.5. If $F$ is a finite subgroup of the mapping class group of a non-sporadic surface, then the limit set of the centralizer $C(F)$ is equal to fix $(F)$, the fixed set of $F$ in $\mathcal{L}_{\text {min }}$.

Proof. If $\lambda$ is a filling lamination in $\overline{\text { fix }_{1}(F)}$, then it is $F$-invariant, as it is a limit of $F$-invariant sets of disjoint simple closed curves. Conversely, if $\lambda$ is a $F$-invariant lamination, then $\lambda / F$ is a lamination in $\mathcal{O}$, so is the limit of a sequence of simple closed curves in $\mathcal{O}$. The pre-images of these simple closed curves are $F$-invariant, and hence lie in $\operatorname{fix}_{1}(F)$, so $\lambda$ is also a limit point of fix $_{1}(F)$. So $\overline{\operatorname{fix}_{1}(F)} \subset \partial \mathcal{C}(\Sigma)$ is equal to the $F$-invariant filling laminations.

The stabilizer of a fixed point set is the subgroup of $G$ which leaves the fixed point set invariant, but does not necessarily fix it pointwise. We now show that the stabilizer of these fixed sets is the normalizer $N(F)$, which is also equal to the $F$-equivariant subgroup. 
Proposition 2.6. Let $F$ be a finite subgroup of $G$. Then the following subgroups of the mapping class group are equal: the $F$-equivariant subgroup $G_{F}$, the normalizer $N(F)$, the stabilizer of $f\left(x_{\mathcal{T}}(F)\right.$ and the stabilizer of $f\left(x_{\partial}(F)\right.$.

Proof. Maclachlan and Harvey [MH75] and Birman and Hilden [BH73, show that the $F$-equivariant subgroup of $G$ is equal to $N(F)$, the normalizer of $F$, and furthermore, Maclachlan and Harvey [MH75] show that the stabilizer of $\operatorname{fix}_{T}(F)$ in $G$ is also equal to $N(F)$, as long as $\operatorname{fix}_{\mathcal{T}}(F)$ is nonempty, which is always the case by the Nielsen realization theorem, due to Kerckhoff Ker83.

The mapping class group $G$ acts continuously on both $\mathcal{T}(\Sigma) \cup \mathcal{P} \mathcal{M L}$ and $\mathcal{C}(\Sigma) \cup \partial \mathcal{C}(\Sigma)$, and the Gromov boundary is the set of filling laminations $\mathcal{L}_{\text {min }}$ which is a dense subset of $\mathcal{P} \mathcal{M L}$. The set of fixed points for $F$ in $\mathcal{T}(\Sigma)$ is an isometrically embedded copy of the Teichmüller space $\mathcal{T}(\mathcal{O})$ for the quotient orbifold, with limit set consisting of the laminations in $\mathcal{P} \mathcal{M L}$ fixed by $F$. Therefore a mapping class group element preserves $\operatorname{fix}_{\mathcal{T}}(F)$ if and only if it preserves $\operatorname{fix}_{\partial}(F)$, so the stabilizer of $\operatorname{fix}_{\partial}(F)$ is equal to the stabilizer of $\operatorname{fix}_{\mathcal{T}}(F)$.

In fact, the stabilizer of $\operatorname{fix}_{\mathcal{T}}(F)$ in the isometry group of $\mathcal{T}(\Sigma)$ is also equal to $N(F)$, as $\operatorname{Isom}(\mathcal{T}(\Sigma))$ is equal to $G$ by Royden's theorem Roy71, see Earle and Kra EK74 for the case of surfaces with punctures.

Proposition 2.7. Let $g$ be an element of the the mapping class group of infinite order. Then the limit set of the centralizer $C(g)$ in the Gromov boundary $\partial \mathcal{C}(\Sigma)$ consists of at most two points.

Proof. If $g$ is pseudo-Anosov, then the centralizer of $g$ is virtually cyclic, and its image in the relative space $\widehat{G}$ is a quasi-geodesic with endpoints consisting of a pair of points in $\mathcal{L}_{\min }$, namely the stable and unstable laminations of $g$.

If $g$ is reducible, then consider a power of $g$ which is pure, i.e. there is a collection of disjoint simple closed curves $a_{i}$, each of which is fixed by $g^{n}$, and $g^{n}$ acts on each complementary subsurface as either the identity or as a pseudo-Anosov. As the centralizer $C(g)$ is contained in the centralizer $C\left(g^{n}\right)$, it suffices to consider the case in which $g$ is pure. We now show that the fixed point set of a reducible pure element $g$ has bounded diameter in the relative metric. If there is a subsurface on which $g$ acts as a pseudo-Anosov, then each curve distance two from any $a_{i}$ has infinitely many images under powers of $g$, so the fixed set of $g$ in $\mathcal{C}(\Sigma)$ has bounded diameter. If $g$ acts as the identity on each complementary subsurface, then $g$ must act as a power of a Dehn twist on one of the fixed curves $a_{i}$. Again, any curve distance two or more from $a_{i}$ has infinitely many images under powers of $g$, and so the fixed set of $g$ has bounded diameter in this case as well. The fixed set of $g$ is preserved by $C(g)$, as if $x \in \mathrm{fix}_{0}(g)$, and $h \in C(g)$ then $g h(x)=h g(x)=h(x)$, so $h(x) \in \operatorname{fix}_{0}(g)$. Furthermore, the image of $C(g)$ in the relative space $\widehat{G}$ also has bounded diameter, as the image of $h$ in $\mathcal{C}(\Sigma)$ is $h\left(x_{0}\right)$. As the fixed set fix $x_{0}(g)$ is preserved by $h$, the distance from $h\left(x_{0}\right)$ to the fixed set is the same as the distance from $x_{0}$ to the fixed set, so $d_{\mathcal{C}}\left(h\left(x_{0}\right)\right.$, fix $\left.x_{0}(g)\right)$ is independent of $h \in C(g)$. This implies that the diameter of $C(g)$ is bounded in the relative metric, and so the limit set of $C(g)$ in the Gromov boundary $\mathcal{L}_{\text {min }}$ is empty.

This implies that if $H$ is a non-elementary subgroup of the mapping class group, and its limit set is contained in the centralizer $C(F)$ of some subgroup $F$ of the mapping class group, then every element of $F$ is finite, and so $F$ is a finite subgroup of the mapping class group.

Proposition 2.8. Let $H$ be a non-elementary subgroup whose limit set is contained in the limit set of the centralizer of a finite subgroup $F$ in the mapping class group $G$. Then $H$ is contained in the normalizer $N(F)$. 
Proof. We may assume that $F$ is a maximal subgroup such that $\bar{H} \subset \overline{C(F)}$, as $F$ is finite, and there is an upper bound on the size of any finite subgroup of $G$, which depends on the surface $\Sigma$. The mapping class group acts continuously on $\mathcal{C}(\Sigma) \cup \partial \mathcal{C}(\Sigma)$, and $\bar{H}$ is $H$-invariant, therefore $h \bar{H} \subset h \overline{C(F)}$ for any $h \in H$. In particular,

$$
\bar{H} \subset \bigcap_{h \in H} h \overline{C(F)} .
$$

A translate $h \overline{C(F)}$ is equal to $\overline{C\left(h F h^{-1}\right)}$, and the intersection of two centralizers $C\left(F_{1}\right)$ and $C\left(F_{2}\right)$ is equal to the centralizer of the group generated by $F_{1} \cup F_{2}$. Therefore $\bar{H}$ is contained in $\overline{C\left(F^{\prime}\right)}$, where $F^{\prime}$ is the group generated by all conjugates of $F$ by elements of $H$. The limit set of $\overline{C\left(F^{\prime}\right)}$ is the intersection of the limit sets $\overline{C(f)}$ of each element $f \in F^{\prime}$. If any element $f$ in $F^{\prime}$ were infinite order, then by Proposition 2.7 the limit set $\overline{C(F)}$ would consist of at most two points. As $H$ is non-elementary, its limit set $\bar{H}$ contains infinitely many points, so all elements of $F^{\prime}$ are finite order, and so $F^{\prime}$ is a finite subgroup of the mapping class group $G$. However, $F^{\prime}$ contains $F$, so by the maximality of $F$, the subgroup $F^{\prime}$ is in fact equal to $F$. This implies that $F$ is invariant under conjugation by elements of $H$, i.e. $H$ is contained in the normalizer $N(F)$, as required.

Let $H^{+}$be a semi-group in $G$, which generates a non-elementary subgroup of the mapping class group, and let $F$ be a finite subgroup of the mapping class group. We now show that either $\overline{C(F)}$ has infinitely many distinct, though not necessarily disjoint, images in $\mathcal{L}_{\min }$ under $H^{+}$, or else $H^{+}$ is contained in the normalizer of $F$.

Proposition 2.9. Let $H^{+}$be a semi-group in the mapping class group which generates a nonelementary subgroup $H$. Then for any finite subgroup $F$ of the mapping class group then either there are infinitely many images of $\overline{C(F)}$ under $H^{+}$, or else $H$ is contained in the normalizer $N(F)$.

Proof. Let $H^{+}$be a semi-group in $G$ which generates a non-elementary subgroup. Suppose there is a finite group $F$ such that there are only finitely many images of $\overline{C(F)}$ under $H^{+}$. There are only finitely many conjugacy classes of finite subgroups in the mapping class group, so we may assume we have chosen a maximal finite subgroup $F$ with this property. Each element of $H^{+}$acts as a finite permutation on the $H^{+}$-orbit of $\overline{C(F)}$, which implies that the inverse of each element in $H^{+}$also acts as a finite permutation on the $H^{+}$-orbit of $\overline{C(F)}$. As $H$ is generated by $H^{+}$, together with the inverses of elements in $H^{+}$, the entire group $H$ acts as finite permutations on the $H^{+}$-orbit of $\overline{C(F)}$. This implies that $H$ contains a finite index subgroup $H^{\prime}$ that preserves $\overline{C(F)}$. As the stabilizer of $\overline{C(F)}$ is the normalizer $N(F)$, by Proposition 2.6, this implies that $H^{\prime}$ is a subgroup of $N(F)$. Therefore the limit set of $H^{\prime}$ is contained in the limit set of $N(F)$. As the limit set of a finite index subgroup is the same as the limit set of the original group, this implies that the limit set of $H$ is contained in the limit set of $C(F)$. Therefore, by Proposition 2.8, $H$ is contained in the normalizer $N(F)$.

\section{Relative conjugacy bounds}

In this section we show that if two elements of the mapping class group $G$ are conjugate, then the relative length of the shortest conjugating word is bounded in terms of the relative lengths of the two conjugate elements. 
Theorem 3.1. Let $a$ and $b$ be conjugate elements of the mapping class group of a non-sporadic surface. Then there is a conjugating word $w$ of relative length $|\widehat{w}| \leqslant K(|\widehat{a}|+|\widehat{b}|)$, for some constant $K$ which only depends on the surface $\Sigma$.

The proof of this result relies on the fact that there is a "discreteness" for the action of the mapping class group on the complex of curves. This means that pseudo-Anosov elements, which act on the complex of curves as hyperbolic isometries, have a minimal translation length, and reducible or periodic isometries, which act as elliptic isometries, act coarsely transitively on their coarse fixed sets.

We will prove this by considering each of the three different types of elements of the mapping class group in turn. Each case will produce a different constant, but we will then choose $K$ to be the maximum of the three constants. Without loss of generality, we may choose $K$ to be at least 1 , and we shall do this, as it will enable us to simplify expressions in subsequent sections.

\subsection{Pseudo-Anosov elements}

Masur and Minsky have shown that the mapping class group has (non-relative) conjugacy bounds for pseudo-Anosov elements, i.e. if a $a$ and $b$ are conjugate pseudo-Anosov elements, then there is a conjugating element $w$ such that $|w| \leqslant K(|a|+|b|)$. Although, a priori, the properties of having relative or non-relative conjugacy bounds for a weakly relatively hyperbolic group may be independent, our argument for the pseudo-Anosov case is clearly modelled on Masur and Minsky's argument from [MM00, and in fact the relative case is substantially simpler.

Pseudo-Anosov elements act on the complex of curves as hyperbolic isometries. A hyperbolic isometry $h$ of a $\delta$-hyperbolic space has a quasi-axis, which is a bi-infinite quasi-geodesic $\alpha$, such that $\alpha$ and $h^{k} \alpha$ are $2 \delta$ fellow travellers for all $k$. In the case of the complex of curves, Masur and Minsky MM00 have shown that we may choose the quasi-axis to be a geodesic, so we will do this, and we will refer to it as an axis for $h$.

The following theorem of Masur and Minsky [MM99] shows that there is a lower bound on the translation length of a pseudo-Anosov element acting on the complex of curves, which only depends on the surface $\Sigma$.

Theorem 3.2. MM99, Proposition 3.6] Let $h$ be a pseudo-Anosov element of the mapping class group of a surface which is not a sphere with three or fewer punctures. Then there is a constant c such that $d_{\mathcal{C}}\left(x, h^{n} x\right) \geqslant c|n|$, for all $x \in \mathcal{C}(\Sigma)$. The constant $c$ depends on the surface $\Sigma$, but is independent of the pseudo-Anosov element $h$.

In MM99, Masur and Minsky state this for non-sporadic surfaces, but the result also holds in the case of a torus with one or fewer punctures or a four-punctured sphere.

We first show there is a lower bound for the distance a point $x$ in $\mathcal{C}(\Sigma)$ is moved by $h$, in terms of the translation distance of $h$ along its axis $\alpha$, and the distance of $x$ from the axis.

Lemma 3.3. Let $\alpha$ be an axis for $h$, and let $x$ be an element of the complex of curves, then $d_{\mathcal{C}}(x, \alpha) \leqslant K_{1} d_{\mathcal{C}}(x, h x)$, where $K_{1}=4 \delta / c$.

Proof. Let $y$ be the closest point to $x$ on the axis $\alpha$. Choose an integer $n \geqslant 8 \delta / c$, which only depends on the surface $\Sigma$, such that $d\left(y, h^{n} y\right) \geqslant 8 \delta$, where $\delta$ is the $\delta$-hyperbolicity constant for $\mathcal{C}(\Sigma)$. Consider a geodesic $\left[x, h^{n} x\right]$, which forms a side of a quadrilateral, together with $[x, y],\left[y, h^{n} y\right]$ and $\left[h^{n} x, h^{n} y\right]$. This is illustrated in Figure 3 below. 


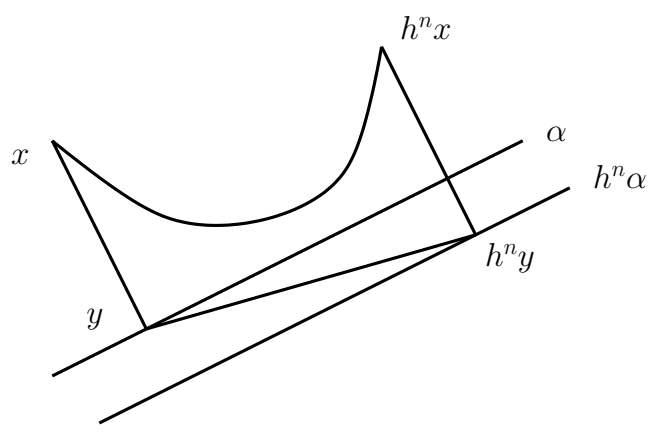

Figure 3: Translation along an axis.

In a $\delta$-hyperbolic space any side of a quadrilateral is contained in a $2 \delta$-neighbourhood of the other three. As $[x, y]$ and $\left[h^{n} x, h^{n} y\right]$ are shortest paths to $\alpha$ and $h^{n} \alpha$, which lie in $2 \delta$ neighbourhoods of each other, any point on $\left[y, h^{n} y\right]$ which is at least $4 \delta$ away from each of its endpoints must also be at least $2 \delta$ away from $[x, y]$ and $\left[h^{n} x, h^{n} y\right]$, so must be $2 \delta$ close to $\left[x, h^{n} x\right]$. So the length of $\left[x, h^{n} x\right]$ must be at least $2 d_{\mathcal{C}}(x, y)+d_{\mathcal{C}}\left(y, h^{n} y\right)-8 \delta \geqslant 2 d_{\mathcal{C}}(x, y)$.

As $d_{\mathcal{C}}\left(x, h^{n} x\right) \leqslant n d_{\mathcal{C}}(x, h x)$, this means $d_{\mathcal{C}}(x, y) \leqslant \frac{n}{2} d_{\mathcal{C}}(x, h x)$, so the claim follows, with $K_{1}=$ $n / 2=4 \delta / c$, which only depends on the surface $\Sigma$, as required.

Now let $a$ and $b$ be two conjugate elements of $G$, and let $w$ be some conjugating element, such that $a=w b w^{-1}$. Let $\alpha$ be an axis for $a$ and let $\beta$ be an axis for $b$. Let $y$ be the closest point in $\alpha$ to $x_{0}$, and let $z$ be the closest point on $\beta$ to $x_{0}$, as illustrated below in Figure 4 .

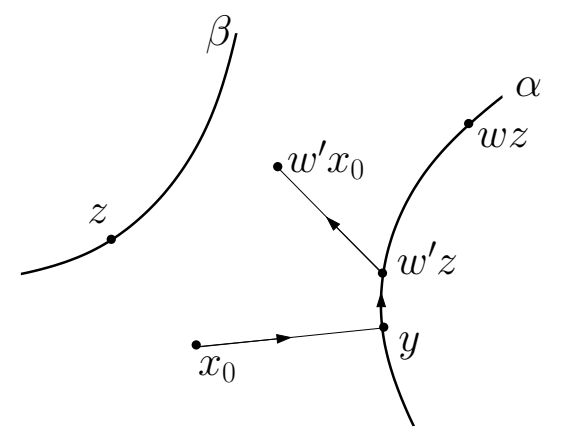

Figure 4: Estimating $\mid \widehat{w^{\prime}}$.

The spaces $\widehat{G}$ and $\mathcal{C}(\Sigma)$ are quasi-isometric, so $\frac{1}{Q}|\widehat{a}| \leqslant d_{\mathcal{C}}\left(x_{0}, a x_{0}\right) \leqslant Q|\widehat{a}|$, where $Q$ is the quasi-isometry constant from (11). Therefore, using Lemma 3.3 above, $d_{\mathcal{C}}\left(x_{0}, y\right) \leqslant Q K_{1}|\widehat{a}|$, and $d_{\mathcal{C}}\left(x_{0}, z\right) \leqslant Q K_{1}|\widehat{b}|$. The conjugating element $w$ takes the axis $\beta$ to the axis $\alpha$, so $w z \in \alpha$. The translation length of $a$ is at most $d_{\mathcal{C}}\left(x_{0}, a x_{0}\right) \leqslant Q|\widehat{a}|$, and so $a$ acts $Q|\widehat{a}|$-coarsely transitively on its axis $\alpha$, so there is a $k$ such that $d_{\mathcal{C}}\left(y, a^{k} w z\right) \leqslant Q|\widehat{a}|$. We can choose to conjugate $b$ to $a$ by $w^{\prime}=a^{k} w$ instead of $w$, and we now show that the relative length of $w^{\prime}$ is bounded. 
The relative length of $w^{\prime}$ is bounded in terms of the distance it moves the base point,

$$
\frac{1}{Q}\left|\widehat{w^{\prime}}\right|-q \leqslant d_{\mathcal{C}}\left(x_{0}, w^{\prime} x_{0}\right)
$$

But this distance is at most the length of the path formed by going from $x_{0}$ to $y \in \alpha$, then along the axis to $w^{\prime} z$, and then to $w^{\prime} x_{0}$, as illustrated above in Figure 4 .

$$
\frac{1}{Q}\left|\widehat{w^{\prime}}\right|-q \leqslant d_{\mathcal{C}}\left(x_{0}, y\right)+d_{\mathcal{C}}\left(y, w^{\prime} z\right)+d_{\mathcal{C}}\left(w^{\prime} z, w^{\prime} x_{0}\right)
$$

The first term is roughly $|\widehat{a}|$, the second term is at most the translation length of $a$, and the final term is equal to $d_{\mathcal{C}}\left(z, x_{0}\right)$, which is roughly $|\widehat{b}|$.

$$
\frac{1}{Q}\left|\widehat{w^{\prime}}\right|-q \leqslant Q K_{1}|\widehat{a}|+Q|\widehat{a}|+Q K_{1}|\widehat{b}|
$$

The only mapping class group element of relative length zero is the identity, so we may assume that both $|\widehat{a}|$ and $|\widehat{b}|$ are at least 1 . Therefore $\left|\widehat{w^{\prime}}\right| \leqslant Q^{2}\left(K_{1}+1\right)|\widehat{a}|+\left(Q^{2} K_{1}+q\right)|\widehat{b}| \leqslant Q^{2}\left(K_{1}+1+q\right)(|\widehat{a}|+|\widehat{b}|)$. So we have shown that for pseudo-Anosov elements we may choose the conjugacy bound constant to be $K=2 Q^{2}\left(K_{1}+1+q\right)$, which only depends on the surface $\Sigma$.

\subsection{Reducible elements}

Reducible elements of the mapping class group act on the complex of curves as elliptic isometries. A reducible element $h$ leaves invariant a collection of disjoint simple closed curves. Following Ivanov Iva92, we say an element of the mapping class group is pure, if there is a disjoint collection of simple closed curves $\sigma(h)$ which are fixed individually by $h$, such that each complementary component of $\sigma(h)$ is also fixed, and furthermore $h$ acts on each complementary component as either a pseudoAnosov element or the identity. If the collection of simple closed curves $\sigma(h)$ has the property that no simple closed curve with non-zero intersection number with $\sigma(h)$ is fixed by $h$, then $\sigma(h)$ is called a canonical reduction system for $h$. If $h$ is not pure, then we define the canonical reduction set $\sigma(h)$ to be the canonical reduction set of some pure power of $h$. Given a reducible element $h$ of the mapping class group, we can raise $h$ to some power $m_{1}$ so that $h^{m_{1}}$ is pure. There is a power $m_{1}$ which works for all reducible elements of the mapping class group, depending only on $\Sigma$.

We start by showing that there is a lower bound on the distance a point is moved by $h$ in terms of its distance from the fixed curves.

Lemma 3.4. Let $h$ be a reducible element of the mapping class group of a non-sporadic surface, with canonical reduction set $\sigma(h)$, and let $y$ be a vertex of the complex of curves. Then $d_{\mathcal{C}}(\sigma(h), y) \leqslant$ $K_{2} d_{\mathcal{C}}(y, h y)+3$, for some constant $K_{2}$, which only depends on the surface $\Sigma$.

Proof. We may raise $h$ to some power $m_{1}$, depending on $\Sigma$, such that $h^{m_{1}}$ is pure, and we shall just write $h$ for $h^{m_{1}}$ from now on.

Given a connected subsurface $\Sigma^{\prime} \subset \Sigma$ with essential boundary components, which is not a threepunctured sphere, Masur and Minsky [MM00] define a subsurface projection $\pi: \mathcal{C}(\Sigma) \rightarrow \mathcal{C}(\Sigma) \cup \varnothing$, which we now describe. Given a simple closed curve $y$ in $\mathcal{C}(\Sigma)$ which intersects $\partial \Sigma^{\prime}$ efficiently, we 
can take a component of $y \cap \Sigma^{\prime}$ and complete it to a simple closed curve in $\Sigma^{\prime}$ by adding a boundary parallel arc. This actually gives a map from $\mathcal{C}(\Sigma)$ to subsets of $\mathcal{C}\left(\Sigma^{\prime}\right)$, but the image has bounded diameter independent of $y$, so this is coarsely well-defined. If $y$ does not intersect $\Sigma^{\prime}$ then we send it to the empty set. In the case where the subsurface is an annulus, Masur and Minsky provide an appropriate definition of a complex for the annulus, which is coarsely equivalent to $\mathbb{Z}$, and which roughly speaking counts how many times the arcs of $y$ wrap around the annulus. We will not give the details of this definition here, as the only property we will use is that a Dehn twist in the core curve of the annulus acts with translation distance one on the complex for the annulus.

We will use the following bounded geodesic image theorem of Masur and Minsky [MM00, which says that a geodesic which is not close to $\partial \Sigma^{\prime} \subset \mathcal{C}(\Sigma)$ projects to a bounded set under the subsurface projection.

Theorem 3.5. MM00, Theorem 3.1] Let $\Sigma^{\prime}$ be an essential connected subsurface of $\Sigma$, which is not a three-punctured sphere, and let $\gamma$ be a geodesic segment in $\mathcal{C}(\Sigma)$, such that $\pi(v) \neq \varnothing$ for every vertex $v \in \gamma$. Then there is a constant $M$, which only depends on $\Sigma$, such that the diameter of $\pi(\gamma)$ is at most $M$.

Suppose there is a component $\Sigma^{\prime}$ of $\Sigma \backslash \sigma(h)$ on which $h$ acts as a pseudo-Anosov element. Then by Theorem 3.2, the translation distance of the pseudo-Anosov element $\left.h\right|_{\Sigma^{\prime}}$ on the complex of curves $\mathcal{C}\left(\Sigma^{\prime}\right)$ is bounded below, so we can raise $h$ to some power $m_{2}$, which only depends on $\Sigma$, so that $h^{m_{2}}$ has translation distance at least $M+1$ on $\mathcal{C}\left(\Sigma^{\prime}\right)$. Otherwise, if there are no components where $h$ acts as a pseudo-Anosov, then there is a simple closed curve $x$ in $\sigma(h)$ such that $h$ acts as a Dehn twist in a neighbourhood of $x$, then choose $\Sigma^{\prime}$ to be a regular neighbourhood of $x$, and then $h^{M+1}$ has translation distance $M+1$ on $\mathcal{C}\left(\Sigma^{\prime}\right)$.

We may assume $h$ acts as the identity in a neighbourhood of the boundary of the surface $\Sigma$. Let $y$ be a simple closed curve in $\mathcal{C}(\Sigma)$, then the subsurface projection map $\pi: \mathcal{C}(\Sigma) \rightarrow \mathcal{C}\left(\Sigma^{\prime}\right)$ only alters $y \cap \Sigma^{\prime}$ in a neighbourhood of the boundary of the subsurface $\Sigma^{\prime}$, so the subsurface projection $\pi$ and $\left.h\right|_{\Sigma^{\prime}}$ commute.

Now let $y$ be a vertex of the complex of curves such that $d_{\mathcal{C}}(\sigma(h), y) \geqslant 3$, and let $\gamma$ be a geodesic in $\mathcal{C}(\Sigma)$ from $y$ to $h y$. If $d_{\mathcal{C}}(\gamma, \sigma(h)) \geqslant 3$, then Theorem 3.5 implies that the image of the projection $\pi(\gamma)$ in $\mathcal{C}(\Sigma)$ has diameter at most $M$. However, $\pi(\gamma)$ contains both $\pi(y)$ and $\pi(h y)=h(\pi(y))$, which are distance at least $M+1$ apart, which gives a contradiction. So $\gamma$ must pass within distance three of $\sigma(h)$, which implies that $d_{\mathcal{C}}(y, h y)$ is at least $2 d_{\mathcal{C}}(\sigma(h), y)-6$.

As we may have raised $h$ to some power, we may take $K_{2}=\frac{1}{2} m_{1} \max \left\{m_{2}, M+1\right\}+6$, which only depends on $\Sigma$. Furthermore, we assumed $d_{\mathcal{C}}(\gamma, \sigma(h)) \geqslant 3$, so we also need an additive term of 3 in the inequality.

Now suppose that $a$ and $b$ are conjugate reducible elements of the mapping class group, with $a=w b w^{-1}$, for some conjugating word $w$. Let $A$ be the canonical reduction set for $a$, and let $B$ be the canonical reduction set for $b$.

By Lemma 3.4 above, $d_{\mathcal{C}}\left(x_{0}, A\right) \leqslant K_{2} d_{\mathcal{C}}\left(x_{0}, a x_{0}\right)+3$, which in turn is at most $Q K_{2}|\widehat{a}|+3$, as $\widehat{G}$ and $\mathcal{C}(\Sigma)$ are $Q$-quasi-isometric. Similarly, $d_{\mathcal{C}}\left(x_{0}, B\right) \leqslant Q K_{2}|\widehat{b}|$. The conjugating element $w$ takes $B$ to $A$, and the diameter of the fixed sets is 2 , so $d_{\mathcal{C}}\left(x_{0}, w x_{0}\right)$ is at most the length of a path from $x_{0}$ to $A$, and then from $A$ to $w x_{0}$, plus 2 . This implies

$$
d_{\mathcal{C}}\left(x_{0}, w x_{0}\right) \leqslant d_{\mathcal{C}}\left(x_{0}, A\right)+d_{\mathcal{C}}\left(A, w x_{0}\right)+2 .
$$


The first term is bounded in terms of $|\widehat{a}|$, and the second term is equal to $d_{\mathcal{C}}\left(B, x_{0}\right)$, as $w B=A$, and so is bounded in terms of $|\widehat{b}|$. Therefore we obtain a bound in terms of the relative lengths of $a$ and $b$,

$$
d_{\mathcal{C}}\left(x_{0}, w x_{0}\right) \leqslant Q K_{2}|\widehat{a}|+Q K_{2}|\widehat{b}|+5
$$

This means that $|\widehat{w}| \leqslant Q^{2} K_{2}(|\widehat{a}|+|\widehat{b}|)+Q(5+q)$. We may assume $|\widehat{a}|$ and $|\widehat{b}|$ have length at least one, so we may choose $K=Q^{2} K_{2}+Q(5+q)$, which only depends on the surface $\Sigma$.

\subsection{Periodic elements}

Periodic elements of the mapping class group act on the complex of curves as elliptic isometries. We now review some useful properties of elliptic isometries, as described for example in Bridson and Haefliger BH99]. In coarse geometry the analogue of the fixed point set of an elliptic isometry of hyperbolic space is the $\epsilon$-fixed set of a periodic element $h$, which is all points moved at most $\epsilon$ by the isometry, i.e. $\operatorname{fix}_{\epsilon}(h)=\left\{x \in \mathcal{C}(\Sigma) \mid d_{\mathcal{C}}(x, h x) \leqslant \epsilon\right\}$. Let $X$ be a bounded set in a $\delta$-hyperbolic space $Y$, with radius $\rho=\inf \left\{\rho \mid X \subset B_{\rho}(x)\right.$ for some $\left.x \in X\right\}$, where $B_{\rho}(x)$ is the ball of radius $\rho$ with center $x$. For $\epsilon>0$ the quasi-center of $X$ is $\operatorname{Center}_{\epsilon}(X)=\left\{y \in Y \mid X \subset B_{\rho+\epsilon}(y)\right\}$.

Lemma 3.6. [BH99, Lemma 3.3] Let $X$ be a bounded set with quasi-center Center $_{\epsilon}(X)$. Then the diameter of Center $\epsilon_{\epsilon}(X)$ is at most $4 \delta+2 \epsilon$.

We shall choose $\epsilon=\delta$, and define the coarse fixed set of a periodic element $h$ to be $\operatorname{fix}_{6 \delta}(h)=$ $\left\{x \in \mathcal{C}(\Sigma) \mid d_{\mathcal{C}}(x, h x) \leqslant 6 \delta\right\}$. The orbit of any point under $h$ is finite, and hence has a quasi-center, which is contained in the coarse fixed set. In particular, the coarse fixed set is non-empty.

Lemma 3.7. Let $H$ be a finite cyclic subgroup of the mapping class group of a non-sporadic surface, generated by a periodic element $h$. Then the centralizer $C(H)$ acts $K_{3}$-coarsely transitively on the coarse fixed set fix $x_{6 \delta}(H)$. Furthermore, for any $x, d_{\mathcal{C}}\left(x, f i x_{6 \delta}(h)\right) \leqslant K_{3} d_{\mathcal{C}}(x, h x)$. The constant $K_{3}$ only depends on the surface $\Sigma$.

Proof. Let $h$ be a periodic element of the mapping class group, with period $n$, and let $H$ be the finite cyclic subgroup generated by $h$. The centralizer $C(H)$ acts coarsely transitively on the 1-fixed set $\operatorname{fix}_{1}(H)$, by Proposition 2.4. We will show that $\operatorname{fix}_{1}(H)$ and $\operatorname{fix}_{K}(H)$ have the same limit sets and are quasiconvex, which implies that $C(H)$ acts coarsely transitively on fix $_{K}(H)$. The fact that the constant in the final inequality only depends on $\Sigma$ then follows from the fact that there is an upper bound on the order of a periodic element of the mapping class group $G$, depending only the surface $\Sigma$.

We now show that $\operatorname{fix}_{1}(H)$ is a quasi-convex subset of $\mathcal{C}(\Sigma)$. The Teichmüller space $\mathcal{T}(\mathcal{O})$, where $\mathcal{O}$ is $\Sigma / H$, can be identified with the fixed set of $H$ in $\mathcal{T}(\Sigma)$, which is isometrically embedded, so Teichmüller geodesics between points in the fixed set of $h$ in $\mathcal{T}(\Sigma)$ are in fact contained in the fixed set of $H$ in $\mathcal{T}(\Sigma)$. Masur and Minsky [MM99] show that Teichmüller geodesics give rise to unparameterized quasi-geodesics in the complex of curves, where an unparameterized quasigeodesic is contained in a uniform neighbourhood of a geodesic, and makes coarsely monotone, but not necessarily coarsely uniform, progress along the geodesic. This means that the map from $\mathcal{C}(\mathcal{O})$ to $\mathcal{C}(\Sigma)$ is a quasiconvex embedding, so the convex hull of $\overline{f i x_{1}(H)}$ is contained in a bounded 
neighbourhood of fix $(H)$. In fact, Rafi and Schleimer [RS09] have shown that this map is a quasiisometric embedding.

We now show that $d_{\mathcal{C}}\left(x, \operatorname{fix}_{6 \delta}(h)\right) \leqslant K_{3} d_{\mathcal{C}}(x, h x)$, for any $x \in C(\Sigma)$. Let $H . x$ be the orbit of $x$ under $H$, and let $\operatorname{Center}_{\delta}(H . x)$ be the quasi-center of H.x. Suppose $y \in \operatorname{Center}_{\delta}(H . x)$, then $H . x \subset B_{\rho+\delta}(y)$, where $\rho$ is the radius of $H . x$, so in particular,

$$
d_{\mathcal{C}}\left(x, \operatorname{Center}_{\delta}(H . x)\right) \leqslant \rho+\delta .
$$

The set $\operatorname{Center}_{\delta}(H . x)$ is $H$-invariant, as $H . x$ is $H$-invariant, and has diameter at most $6 \delta$, so $\operatorname{Center}_{\delta}(H . x) \subset \operatorname{fix}_{6 \delta}(H)$. The diameter of $H . x$ is at most $n d_{\mathcal{C}}(x, h x)$, and so $d_{\mathcal{C}}\left(x, \operatorname{Center}_{\delta}(H . x)\right) \leqslant$

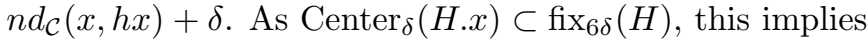

$$
d_{\mathcal{C}}\left(x, \operatorname{fix}_{6 \delta}(H)\right) \leqslant N d_{\mathcal{C}}(x, h x)+\delta,
$$

where $N$ is the maximum period of any periodic element of $G$, which only depends on the surface $\Sigma$. If $d_{\mathcal{C}}(x, h x)$ is zero, then $x$ is fixed by $h$, so $d_{\mathcal{C}}\left(x, \operatorname{fix}_{6 \delta}(H)\right)$ is also zero, so we may choose the constant $K_{3}$ here to be $N+\delta$.

Finally, we show that $\operatorname{fix}_{K}(H)$ is quasiconvex for $K \geqslant 6 \delta$, and $C(H)$ acts coarsely transitively on $\operatorname{fix}_{K}(H)$. Let $a$ and $b$ be points in $\operatorname{fix}_{K}(H)$, then the orbits $H . a$ and $H . b$ each have diameter at most $n K$, and so the geodesics $\left[h^{i} a, h^{i} b\right]$ are $2 \delta$-fellow travellers, outside of $n K$-neighbourhoods of their endpoints, where $n$ is the period of $h$. Therefore, for $x \in[a, b]$, the orbit $H . x$ has diameter at most $n(K+2 \delta)$, and so $d_{\mathcal{C}}\left(x, \operatorname{Center}_{\delta}(H . x)\right) \leqslant n(K+2 \delta)+\delta$. As $\operatorname{Center}_{\delta}(H . x) \subset$ fix $_{6 \delta}(H)$, which in turn is contained in $\operatorname{fix}_{K}(H)$, this implies that every geodesic $[a, b]$ with endpoints in fix $x_{K}(H)$ is contained in a $(N K+2 N \delta+\delta)$-neighbourhood of $\operatorname{fix}_{K}(H)$, where $N$ is the largest order of a periodic element in $G$, which depends only on $\Sigma$. As fix $x_{K}(H)$ is quasiconvex, and has the same limit set as $\operatorname{fix}_{1}(H)$, fix $_{K}(H)$ lies in a bounded neighbourhood of fix $x_{1}(H)$, and so as $C(H)$ acts coarsely transitively on fix $_{1}(H), C(H)$ also acts coarsely transitively on fix $x_{K}(H)$. Therefore, we make take $K_{3}$ to be the maximum of $N+\delta$, and the constant by which $C(H)$ acts coarsely transitively on $\operatorname{fix}_{6 \delta}(H)$.

Let $a$ and $b$ be conjugate periodic elements of the mapping class group, so $a=w b w^{-1}$, for some conjugating word $w$. Let $A$ be the cyclic subgroup generated by $a$, and let $B$ be the cyclic subgroup generated by $b$. Let $y$ be the closest point in $\operatorname{fix}_{6 \delta}(a)$ to $x_{0}$, and let $z$ be the closest point in $\operatorname{fix}_{6 \delta}(b)$ to $x_{0}$. The conjugating element $w$ takes $\operatorname{fix}_{6 \delta}(b)$ to $\operatorname{fix}_{6 \delta}(a)$, so $w z \in \operatorname{fix}_{6 \delta}(a)$. As $C(a)$ acts $K_{3}$-coarsely transitively on $\operatorname{fix}_{6 \delta}(a)$, there is $c \in C(a)$ such that $d_{\mathcal{C}}(y, c w z) \leqslant K_{3}$. As $c$ is in the centralizer of $a$, the element $c w$, which we shall denote $w^{\prime}$, conjugates $b$ to $a$.

We can estimate the relative length of the conjugating element $\left|\widehat{w^{\prime}}\right|$ in terms of the distance in the complex of curves from $x_{0}$ to $w^{\prime} x_{0}$, which is at most the length of the path from $x_{0}$ to $y$, then from $y$ to $w^{\prime} z$, and finally from $w^{\prime} z$ to $w^{\prime} x_{0}$. Figure 4 also illustrates this case, if the axes $\alpha$ and $\beta$ are replaced with the coarse fixed sets $\operatorname{fix}_{6 \delta}(a)$ and $\operatorname{fix}_{6 \delta}(b)$ respectively.

$$
d_{\mathcal{C}}\left(x_{0}, w^{\prime} x_{0}\right) \leqslant d_{\mathcal{C}}\left(x_{0}, y\right)+d_{\mathcal{C}}\left(y, w^{\prime} z\right)+d_{\mathcal{C}}\left(w^{\prime} z, w^{\prime} x_{0}\right)
$$

By Lemma 3.7, the first term on the right hand side is at most $K_{3} d_{\mathcal{C}}\left(x_{0}, a x_{0}\right)$. Similarly the final term on the right hand side is at most $K_{3} d_{\mathcal{C}}\left(x_{0}, b x_{0}\right)$. The middle term is bounded by $K_{3}$.

$$
d_{\mathcal{C}}\left(x_{0}, w^{\prime} x_{0}\right) \leqslant K_{3} d_{\mathcal{C}}\left(x_{0}, a x_{0}\right)+K_{3}+K_{3} d_{\mathcal{C}}\left(x_{0}, b x_{0}\right)
$$


The first term on the right hand side is at most $Q K_{3}|\widehat{a}|$, using the quasi-isometry between $\widehat{G}$ and $\mathcal{C}(\Sigma)$. Similarly, the final term is at most $Q K_{3}|\widehat{b}|$.

$$
d_{\mathcal{C}}\left(x_{0}, w^{\prime} x_{0}\right) \leqslant Q K_{3}|\widehat{a}|+K_{3}+Q K_{3}|\widehat{b}|
$$

Therefore, $\left|\widehat{w^{\prime}}\right| \leqslant Q^{2} K_{3}(|\widehat{a}|+|\widehat{b}|)+Q\left(K_{3}+q\right)$, and as we may assume that $a$ and $b$ have relative length at least 1 , we may take $K=Q^{2} K_{3}+Q\left(K_{3}+q\right)$, which only depends on the surface $\Sigma$.

This completes the proof of Theorem 3.1. The conjugacy bound constants we have obtained in each of the above sections may be different, however, we may choose $K$ to be the maximum such constant for the three types of elements of the mapping class group.

\section{Conjugates of relatively short elements}

In this section we consider collections of elements which are conjugates of relatively short elements, and we investigate how they are distributed inside $G$. The results of this section hold for any group $G$ which is weakly relatively hyperbolic and which has relative conjugacy bounds. We shall refer to the constant of hyperbolicity $\delta$ and the relative conjugacy bound constant $K$ as the group constants. In particular, the results of this section apply to the mapping class group, and the collection of nonpseudo-Anosov elements in the mapping class group consists of elements which are all conjugate to elements of bounded relative length.

Let $R$ be a collection of elements of $G$, which are conjugate to elements of relative length at most $B$. In some parts of $G$, elements of $R$ are close together, in other parts of $G$ they are far apart. We quantify this by defining $R_{k}$ to be the $k$-dense subset of $R$, consisting of all elements of $R$ which are distance at most $k$ in $G$ from some other element of $R$, i.e. $R_{k}=\left\{r \in R \mid\right.$ there is an $r^{\prime} \in$ $R$ with $r \neq r^{\prime}$ and $\left.d\left(r, r^{\prime}\right) \leqslant k\right\}$. This definition uses word length in $G$, not relative length.

Any element in $R_{k}$ differs from another element of $R$ by an element $g$ of word length at most $k$, so in fact $R_{k}$ is the finite union of sets $R \cap R g$, as $g$ runs over all group elements of word length at most $k$. The limit set of $R$, and hence of $R g$, may be the entire boundary, and this is the case, for example, if $R$ consists of all non-pseudo-Anosov elements. However, the limit set of the intersection $\overline{R \cap R g}$ may be smaller than than the intersection of the limit sets $\bar{R} \cap \overline{R g}$. For example, if $R$ and $R g$ are disjoint, then the limit set of their intersection will be empty. In this section we will show that the elements of $R \cap R g$ are contained in a particular neighbourhood of the centralizer of $g$, which we shall call a horoball neighbourhood, as its definition is reminiscent of the definition of a horoball in hyperbolic space. Let $X$ be a subset of $\widehat{G}$, and let $L$ be a constant. We define an $L$-horoball neighbourhood of $X$, which we shall denote $\mathcal{O}_{L}(X)$, to be the union of balls in $\widehat{G}$ centered at $x \in X$, of radius $|\widehat{x}|+L$, i.e.

$$
\mathcal{O}_{L}(X)=\bigcup_{x \in X} \widehat{B}_{|\widehat{x}|+L}(x)
$$

This definition uses relative distance in $\widehat{G}$. The limit set of $\mathcal{O}_{L}(X)$ is the same as the limit set of $X$. This is because if a sequence $y_{n} \in \mathcal{O}_{L}(X)$ converges to the Gromov boundary, then each $y_{n}$ lies in $B_{\left|\widehat{x_{n}}\right|+L}\left(x_{n}\right)$ for some $x_{n} \in X$. If $y_{n}$ and $x_{n}$ limit to distinct points in the Gromov boundary, then the nearest point projection of $y_{n}$ to the geodesic $\left[x_{0}, x_{n}\right]$ stays a bounded distance from $x_{0}$, but this implies that the distance from $y_{n}$ to $x_{n}$ is bounded, a contradiction, see [Mah10, Lemma 3.1] for a more detailed version of this argument. 
Theorem 4.1. Let $G$ be a weakly relatively hyperbolic group with relative conjugacy bounds, and let $R$ be a set of elements which are conjugate to elements of relative length at most B. Then for any element $g$, there is a constant $L$, which only depends on $B$, $|\widehat{g}|$, and the group constants $\delta$ and $K$, such that $R \cap R g$ is contained in an L-horoball neighbourhood of the centralizer of $g$.

This theorem shows that the limit set of $R \cap R g$ is contained in the limit set of the centralizer of $g$, and hence that the limit set of $R_{k}$ is contained in the finite union of limit sets of centralizers of elements of $G$ with word length at most $k$. In the case that $R$ is the set of non-pseudo-Anosov elements, the union of the limit sets of centralizers is dense in the boundary, so the limit set of the union of the $R_{k}$, over all $k$, is the entire boundary, which must be the case, as the limit set of $R$ is the entire boundary.

We start by showing that if $r$ is conjugate to $s$, and the conjugating element $w$ is chosen to be one of shortest relative length, then the path in $\widehat{G}$ corresponding to $w s w^{-1}$ is quasi-geodesic, with quasi-geodesic constants depending only on the relative length of $s$ and the group constants $\delta$ and $K$, and independent of the relative length of $r$.

Lemma 4.2. Let $G$ be a weakly relatively hyperbolic group with relative conjugacy bounds. Let $r$ be an element of $G$ which is conjugate to an element s, i.e. $r=w s w^{-1}$, for some $w \in G$. If we choose $w$ to be a conjugating word of shortest relative length, then the word wsw $w^{-1}$ is quasi-geodesic in $\widehat{G}$, with quasi-geodesic constants which depend only on the relative length of $s$, and the group constants $\delta$ and $K$.

Proof. We will use the fact that in a $\delta$-hyperbolic space, a path is a quasigeodesic if and only if the path lies in a bounded neighbourhood of a geodesic, and the projection of the path onto the geodesic makes linear progress along the geodesic. As the path we will consider is a union of three geodesic segments, one of which has bounded length, it suffices to show that the path is contained in a bounded neighbourhood of a geodesic. If the path $w s w^{-1}$ travels far away from a relative geodesic from 1 to $r$, then as $s$ has bounded relative length, there must be a final subsegment of $w$, and an initial subsegment of $w^{-1}$, which fellow travel. As the path corresponding to $w^{-1}$ is the orientation reverse of $w$, we may choose these initial and final segments to be inverses of each other. This implies there is a long final subword of $w$ which conjugates $s$ to an element of bounded relative length. As the group has relative conjugacy bounds, we can replace this long subsegment of $w$ with a shorter word, contradicting our assumption that $w$ was a conjugating element of shortest relative length. We now write out a detailed version of this argument.

We will label relative geodesics by their endpoints, so we will write $[1, w]$ for a particular choice of relative geodesic from 1 to $w$. Relative geodesics are in general not unique, but in fact we will not need to refer to multiple relative geodesics with the same endpoints. A relative geodesic is a word in the mapping class group, and we will write $[1, w]^{-1}$ to denote the inverse of this word, which is a relative geodesic from 1 to $w^{-1}$. We may also think of the path $[1, w]^{-1}$ as a translate of $[1, w]$, but with the reverse orientation. We will also choose relative geodesics $[1, s]$ and $[1, r]$ from 1 to $s$ and from 1 to $r$ respectively. The group acts on $\widehat{G}$ on the left by isometries, so $w[1, s]$ is a relative geodesic from $w$ to $w s$. In particular, the union of the three relative geodesics $[1, w], w[1, s]$ and $w s[1, w]^{-1}$, is a path from 1 to $r$, which corresponds to the concatenation of the words chosen to represent $w, s$ and $w^{-1}$. This is illustrated below in Figure 5. Note that the segment $w s[1, w]^{-1}$ is equal to the segment $r[1, w]$, but with the reverse orientation. 


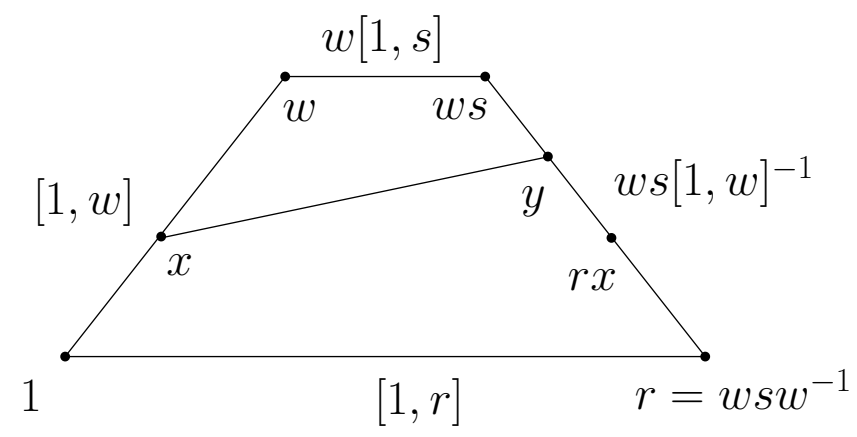

Figure 5: The word corresponding to $w s w^{-1}$ gives a path from 1 to $r$.

We may assume that $[1, r]$ and $w[1, s]$ are reasonably far apart. By thin triangles, the geodesics $[1, w]$ and $w s\left[1, w^{-1}\right]$ have long subsegments which fellow travel. This implies there is a subsegment of $[1, w]$ which conjugates $s$ to a short word, and as the group has relative conjugacy bounds, this implies that we may choose $w$ to have bounded relative length. We now fill in the details of this argument.

Let $D$ be the relative distance from $w[1, s]$ to $[1, r]$. If $D \leqslant|\widehat{s}|+4 \delta+2$, then the entire path $[1, w] \cup w[1, s] \cup w s[1, w]^{-1}$ is contained in a $2|\widehat{s}|+6 \delta+2$ neighbourhood of $[1, r]$, so assume this is not the case. A group element $x$ lying on $[1, w]$ divides the segment into an initial segment from 1 to $x$, which we shall denote $[1, x]$, and a final segment from $x$ to $w$, which we shall denote $[x, w]$. Choose $x$ to be the group element of shortest relative length such that both $[x, w]$ and $r[x, w]$ lie outside a $2 \delta+1$ neighbourhood of $[1, r]$. Such an element $x$ exists as the distance from $[1, r]$ to $w[1, s]$ is greater than $2 \delta+1$. Note that the distance from $x$ to $w[1, s]$ is also greater than $|\widehat{s}|+2 \delta+1$.

We now show that the distance from $x$ to $r x$ is at most $|\widehat{s}|+4 \delta$. In a geodesic quadrilateral in a $\delta$-hyperbolic space, each edge is contained in a $2 \delta$ neighbourhood of the other three, and as $x$ is distance greater than $2 \delta$ from either $w[1, s]$ or $[1, r]$, this implies that $x$ lies in a $2 \delta$ neighbourhood of the remaining side $w s[1, w]^{-1}$. Let $y$ be the closest point on $w s[1, w]^{-1}$ to $x$. By the triangle inequality, the relative distance from $y$ to $w s$ is at most the relative length of the path from $y$ to $w s$ via $x$ and $w$.

$$
\widehat{d}(y, w s) \leqslant \widehat{d}(y, x)+\widehat{d}(x, w)+\widehat{d}(w, w s)
$$

The distance between $x$ and $y$ is at most $2 \delta$, the relative distance from $x$ to $w$ is the same as the relative distance from $r x$ to $w s$, and the distance between $w$ and $w s$ is $|\widehat{s}|$.

$$
\widehat{d}(y, w s) \leqslant 2 \delta+\widehat{d}(r x, w s)+|\widehat{s}|
$$

Similarly, the relative distance from $x$ to $w$ is at most the relative length of the path from $x$ to $w$ through $y$ and $w s$.

$$
\widehat{d}(x, w) \leqslant \widehat{d}(x, y)+\widehat{d}(y, w s)+\widehat{d}(w s, w)
$$


The relative distance from $x$ to $w$ is the same as the relative distance from $r x$ to $w s$, the relative distance between $x$ and $y$ is at most $2 \delta$, and the length of $\widehat{d}(w s, w)$ is at most $|\widehat{s}|$.

$$
\widehat{d}(r x, w s) \leqslant 2 \delta+\widehat{d}(y, w s)+|\widehat{s}|
$$

Together, lines (1) and (2) imply that the difference between the relative distance of $r x$ from $w s$, and the relative distance of $y$ from $w s$, is at most $2 \delta+|\widehat{s}|$. As $r x$ and $y$ lie on a common relative geodesic through $w s$, this implies that $y$ and $r x$ are relative distance at most $|\widehat{s}|+2 \delta$ apart, and hence that $x$ and $r x$ are relative distance at most $|\widehat{s}|+4 \delta$ apart.

The path composed of the three relatively geodesic segments $[x, w], w[1, s]$ and $r[w, x]$ gives a word in the mapping class corresponding to $\left(x^{-1} w\right) s\left(x^{-1} w\right)^{-1}$. The relative length of this group element is the distance between $x$ and $r x$, which is at most $|\widehat{s}|+4 \delta$, so $x^{-1} w$ conjugates $s$ to a word of length at most $|\widehat{s}|+4 \delta$. As we have assumed that the group $G$ has relative conjugacy bounds, we may choose the relative length of the conjugating element $x^{-1} w$ to be at most $K(2|\widehat{s}|+4 \delta)$, where $K$ is the relative conjugacy bound constant. This implies that distance $D$ between $w[1, s]$ and $[1, r]$ is at most $K(|\widehat{s}|+2 \delta)+2 \delta+1$. Hence the union of the segments $[1, w], w[1, s]$ and $w s[1, w]^{-1}$ lies in an $L_{1}$-neighbourhood of $[1, r]$, where $L_{1}=\max \{K(|\widehat{s}|+2 \delta)+2 \delta+1+|\widehat{s}|, 2|\widehat{s}|+4 \delta+2\} \leqslant 2 K(|\widehat{s}|+2 \delta)+2$. The constant $L_{1}$ depends only on $|\widehat{s}|$, and the group constants $\delta$ and $K$.

We now observe that if $r$ is conjugate to $s$ by a word $w$ of shortest relative length, then the relative length of $w$ is roughly half the relative length of $r$.

Proposition 4.3. Let $G$ be a relatively hyperbolic group. Let $r$ in $G$ be conjugate to $s$ by $w$, such that $w$ and $w s$ are contained in an $L_{1}$-neighbourhood of a relative geodesic $[1, r]$. Then

$$
\frac{1}{2}(|\widehat{r}|-|\widehat{s}|) \leqslant|\widehat{w}| \leqslant \frac{1}{2}(|\widehat{r}|+|\widehat{s}|)+2 L_{1} .
$$

Proof. By the triangle inequality $|\widehat{r}| \leqslant 2|\widehat{w}|+|\widehat{s}|$, giving the left hand inequality. Let $a$ be the closest point on $[1, r]$ to $w$, and let $b$ be the closest point on $[1, r]$ to $w s$. Relative geodesics $[w, a]$ and $[w s, b]$ have length at most $L_{1}$. Let $[1, a]$ be the initial segment of $[1, r]$ from 1 to $a$, and let $[b, r]$ be the terminal segment of $[1, r]$ from $b$ to $r$. The overlap of $[1, a]$ and $[b, r]$ is at most the relative length of $[a, b]$, which is at most $2 L_{1}+|\widehat{s}|$, giving $|\widehat{a}|+\widehat{d}(b, r) \leqslant|\widehat{r}|+2 L_{1}+|\widehat{s}|$. Using the triangle inequality, $|\widehat{w}| \leqslant|\widehat{a}|+L_{1}$ and $|\widehat{w}| \leqslant \widehat{d}(b, r)+L_{1}$, which implies $2|\widehat{w}| \leqslant|\widehat{r}|+4 L_{1}+|\widehat{s}|$. This gives the right hand inequality.

We can now complete the proof of Theorem 4.1

Proof. Suppose $r^{\prime}=r g$, and $r$ and $r^{\prime}$ are conjugate to relatively short words $s$ and $s^{\prime}$ respectively. By Lemma 4.2 we may choose shortest conjugating words $w$ and $w^{\prime}$ so that the paths $w s w^{-1}$ and $w^{\prime} s^{\prime} w^{-1}$ are quasigeodesic, with endpoints close together. This implies that $w$ and $w^{\prime}$ are close together, and as the relative lengths of $s$ and $s^{\prime}$ are bounded, this implies that $w^{-1}$ conjugates $g$ to another short word $w^{-1} g w$, and so $w$ is close to the centralizer of $g$. This in turn implies that $r$ lies in a horoball neighbourhood of the centralizer $C(g)$. We now give a detailed version of this argument.

It suffices to prove the result for elements $r \in R \cap R g$ with $|\widehat{r}|$ sufficiently large. If $r$ is in $R \cap R g$, then there is an $r^{\prime} \in R$ such that $r^{-1} r^{\prime}=g$. Let $[1, r]$ be a relative geodesic from 1 to $r$, and let $\left[1, r^{\prime}\right]$ be a relative geodesic from 1 to $r^{\prime}$. By thin triangles, the two relative geodesics $[1, r]$ and $\left[1, r^{\prime}\right]$ 
are relative distance at most $\delta$ apart for initial segments of relative length at least $|\widehat{r}|-|\widehat{g}|-\delta$. Later on in this argument we will need this to be be at least $\frac{1}{2}\left(|\widehat{r}|+4 L_{1}+B\right)+L_{1}+\delta$, where $L_{1}$ is the constant from Lemma 4.2 above, so we will assume that $|\widehat{r}|>6 L_{1}+B+4 \delta+2|\widehat{g}|$. In particular, this implies that $L$ must be at least as large as this value.

Let $w$ be a word of shortest relative length conjugating $r$ to an element $s$ of relative length at most $B$, and let $w^{\prime}$ be a word of shortest relative length conjugating $r^{\prime}$ to a word $s^{\prime}$ of relative length at most $B$. Choose relative geodesics $[1, w]$ and $[1, s]$ from 1 to $w$ and $s$ respectively. Then the union of the three relatively geodesic segments $[1, w] \cup w[1, s] \cup w s[1, w]^{-1}$ is a path from 1 to $r=w s w^{-1}$. Similarly, choose relative geodesics $\left[1, w^{\prime}\right]$ and $\left[1, s^{\prime}\right]$ from 1 to $w^{\prime}$ and $s^{\prime}$ respectively. Then the union of the three relatively geodesic segments $\left[1, w^{\prime}\right] \cup w^{\prime}\left[1, s^{\prime}\right] \cup w^{\prime} s^{\prime}\left[1, w^{\prime}\right]^{-1}$ is a path from 1 to $r^{\prime}=w^{\prime} s^{\prime} w^{\prime-1}$. This is illustrated below in Figure 6.

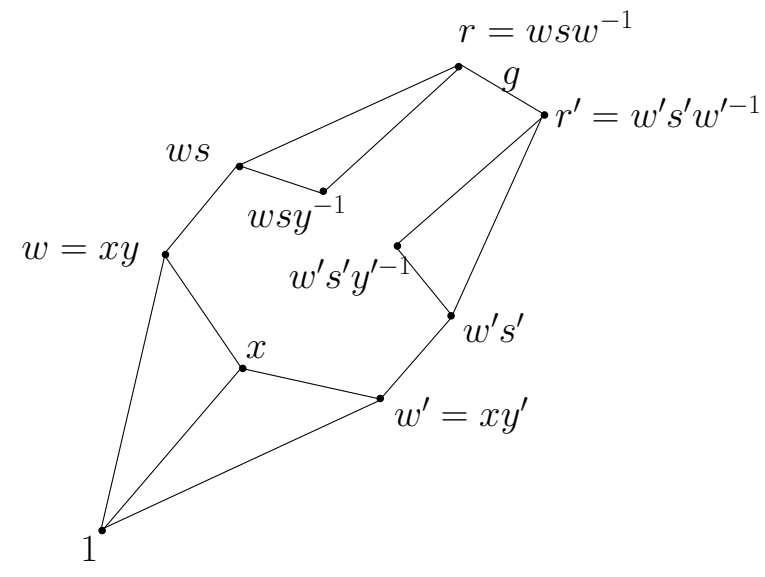

Figure 6: The group elements $r$ and $r^{\prime}$ are conjugate to $s$ and $s^{\prime}$ respectively.

We now show that the two group elements $w$ and $w^{\prime}$ are close together. By Proposition 4.3 , the relative length of $w$ is roughly half that of $r$, i.e. $\frac{1}{2}(|\widehat{r}|-B) \leqslant|\widehat{w}| \leqslant \frac{1}{2}\left(|\widehat{r}|+4 L_{1}+B\right)$, and there are similar bounds for $w^{\prime}$. By Lemma 4.2 the group element $w$ lies in an $L_{1}$-neighbourhood of the geodesic $[1, r]$. Let $a$ be the closest point on $[1, r]$ to $w$, then the relative distance between $w$ and $a$ is at most $L_{1}$, so $\frac{1}{2}(|\widehat{r}|-B)-L_{1} \leqslant|\widehat{a}| \leqslant \frac{1}{2}\left(|\widehat{r}|+4 L_{1}+B\right)+L_{1}$. Again, by Lemma 4.2, the group element $w^{\prime}$ lies in an $L_{1}$-neighbourhood of $\left[1, r^{\prime}\right]$, which, as we have assumed that $|\widehat{r}|$ is sufficiently large, has an initial segment of at least this length contained in a $\delta$ neighbourhood of $[1, r]$. So if $a^{\prime}$ is the closest point on $[1, r]$ to $w^{\prime}$, then the distance from $a^{\prime}$ to $w^{\prime}$ is at most $L_{1}+\delta$. This implies that $\frac{1}{2}(|\widehat{r}|-B)-L_{1}-\delta \leqslant\left|\widehat{a^{\prime}}\right| \leqslant \frac{1}{2}\left(|\widehat{r}|+4 L_{1}+B\right)+L_{1}+\delta$. Therefore, as $a$ and $a^{\prime}$ lie on a common relative geodesic through 1 , the relative distance between $a$ and $a^{\prime}$ is at most $4 L_{1}+B+\delta$, so the relative distance between $w$ and $w^{\prime}$ is at most $6 L_{1}+2 B+2 \delta$.

As $w$ and $w^{\prime}$ are close together, we may choose words to represent them that have a large common initial segment consisting of a relative geodesic $[1, x]$. The relative distance between $w$ and $w^{\prime}$ is at most $6 L_{1}+2 B+2 \delta$, so we may choose a group element $x$ such that $w=x y$ and $w^{\prime}=x y^{\prime}$, for $y$ and $y^{\prime}$ of relative length at most $3 L_{1}+B+\delta$. The path from $w s$ to $r$ gives a word corresponding to $w^{-1}$; in fact this path is precisely $w s[1, w]^{-1}$. As $w^{-1}=y^{-1} x^{-1}$, we may also travel from $w s$ to $r$ using the path $w s[1, y]^{-1} \cup w s y^{-1}[1, x]^{-1}$. Similarly, the path from $w^{\prime} s^{\prime}$ to $r^{\prime}$ is $w^{\prime} s^{\prime}\left[1, w^{\prime}\right]^{-1}$, so we 
may also travel from $w^{\prime} s^{\prime}$ to $r^{\prime}$ along the path $w^{\prime} s^{\prime}\left[1, y^{\prime}\right]^{-1} \cup w^{\prime} s^{\prime} y^{\prime-1}[1, x]^{-1}$. This is illustrated in Figure 6 above.

We now show that the group element $x$ conjugates $g$ to an element of bounded relative length. The two group elements $w s y^{-1}$ and $w^{\prime} s^{\prime} y^{\prime-1}$ are endpoints of a path consisting of three relatively geodesic segments passing through $r$ and $r^{\prime}$, namely $w s y^{-1}[1, x]^{-1} \cup r[1, g] \cup r^{\prime}[1, x]$. The group element corresponding to this path is $x^{-1} g x$, which is a conjugate of $g$, and we now show that the relative length of this element is bounded, i.e. we show that the endpoints of the path are a bounded relative distance apart. The relative distance between $w$ and $w^{\prime}$ is at most $6 L_{1}+2 B+2 \delta$. The group elements $s$ and $s^{\prime}$ have relative length at most $B$, so the distance between $w s$ and $w^{\prime} s^{\prime}$ is at most $6 L_{1}+4 B+2 \delta$. The elements $y$ and $y^{\prime}$ have length at most $3 L_{1}+B+\delta$, so the relative distance between $w s y^{-1}$ and $w s^{\prime} y^{-1}$ is at most $12 L_{1}+6 B+4 \delta$.

Finally, we show that $w$ lies close to the centralizer of $g$, which will imply that $r$ lives in a horoball neighbourhood of $C(g)$. As we have assumed that the group $G$ has relative conjugacy bounds, there is a group element $v$ of length at most $K\left(|\widehat{g}|+12 L_{1}+6 B+4 \delta\right)$ which conjugates $x^{-1} g x$ to $g$, where $K$ is the relative conjugacy bound constant. This implies that $x^{-1} g x=v g v^{-1}$. Multiplying on the right by $v$ and on the left by $x$, we obtain $g x v=x v g$, so $x v$ lies in the centralizer of $g$. Therefore $x$ is a distance at most $K\left(|\widehat{g}|+12 L_{1}+6 B+4 \delta\right)$ from $C(g)$, and so $w$ is a distance at most $L_{2}=K\left(|\widehat{g}|+12 L_{1}+6 B+4 \delta\right)+3 L_{1}+B+\delta$ from $C(g)$. Let $b$ be the closest point in $C(g)$ to $w$. The triangle inequality implies that $|\widehat{w}| \leqslant|\widehat{b}|+L_{2}$. The distance from $w s w^{-1}$ to $C(g)$ is at most $|\widehat{w}|+B+L_{2}$, which is at most $|\widehat{b}|+2 L_{2}+B$. Therefore $w s w^{-1}$ lies in an $L$-horoball neighbourhood of $C(g)$, where $L=2 L_{2}+B$. The constant $L$ depends only on $B,|\widehat{g}|$, and the group constants $\delta$ and $K$.

This completes the proof of Theorem 4.1 .

\section{Random walks}

We start by recalling some basic definitions about random walks on groups, see for example Woess Woe00. We then show that a random walk on the mapping class group converges to a projective measured lamination with probability one, and observe that the periodic and reducible elements are all conjugate to elements of bounded relative length, for some bound which depends on the surface. Then in Section 5.1 we show that the limit set of the centralizer of an element of the mapping class group has harmonic measure zero, as long as the limit set has infinitely many images under the group $H$ generated by the support of the random walk. Let $R$ be a set of elements of $G$ which are conjugate to elements of bounded relative length, and let $R_{k}$ be the set of $k$-dense elements of $R$. We showed in Section 4 that the limit set of $R_{k}$ is contained in a finite union of limit sets of non-trivial centralizers, so this implies that the limit set of $R_{k}$ has harmonic measure zero, under the assumption on the images of limit sets of centralizers. Finally, in Section 5.2 we argue that this means a sample path travels through regions where the elements of $R$ become further and further apart, so the probability that the random walk is in $R$ tends to zero asymptotically. We finally complete the proof by showing how to reduce the general case to the case in which all limit sets of centralizers of non-trivial elements have infinitely many images under $H$.

Let $G$ be a group, and let $\mu$ be a probability distribution on $G$, which we shall call the step distribution. We may use the probability distribution $\mu$ to generate a Markov chain, or random walk on $G$, with transition probabilities $p(x, y)=\mu\left(x^{-1} y\right)$, and we shall always assume that we 
start at time zero at the identity. We shall write $p^{(n)}(x, y)$ for the probability that you go from $x$ to $y$ in $n$ steps. The step space for the random walk is the infinite product $(G, \mu)^{\mathbb{Z}_{+}}$, i.e. the steps or increments of the random walk are a sequence of independent identically $\mu$-distributed random variables. An element of the step space $\left(s_{1}, s_{2}, s_{3}, \ldots\right)$ determines a path in $G$, where the location $w_{n}$ of the path at time $n$ is given by the product of the first $n$ steps, i.e. $w_{n}=s_{1} s_{2} \ldots s_{n}$. This gives a map $\rho: G^{\mathbb{Z}_{+}} \rightarrow G^{\mathbb{Z}_{+}}$from steps to paths, and we will write $\left(G^{\mathbb{Z}_{+}}, \mathbb{P}_{\mu}\right)$ for the path space, where $\mathbb{P}_{\mu}(X)$ is the measure of $\rho^{-1}(X)$ in the step space. We will often just write $\mathbb{P}$ for $\mathbb{P}_{\mu}$ if it is clear from context which probability distribution determines the random walk. We will call an element $\omega=\left(w_{1}, w_{2}, \ldots\right)$ of the path space a sample path. The distribution of random walks at time $n$ is given by the $n$-fold convolution of $\mu$, which we shall write as $\mu^{(n)}$. We shall always require that the group generated by the support of $\mu$ is non-elementary. We do not assume that the probability distribution $\mu$ is symmetric, so the group generated by the support of $\mu$ may be strictly larger than the semi-group generated by the support of $\mu$.

A homomorphism $\phi: G \rightarrow H$ takes random walks on $G$ to random walks on $H$. More precisely, the homomorphism $\phi$ determines a map, which we shall also call $\phi$, from $G^{\mathbb{Z}_{+}}$to $H^{\mathbb{Z}_{+}}$, which sends a sequence $\left(s_{1}, s_{2}, s_{3}, \ldots\right)$ to the sequence $\left(\phi\left(s_{1}\right), \phi\left(s_{2}\right), \phi\left(s_{3}\right), \ldots\right)$. Let $\phi^{*} \mu$ be the probability distribution on $H$ defined by $\phi^{*} \mu(h)=\mu\left(\phi^{-1}(h)\right)$, then $\phi$ gives a measure preserving map from $(G, \mu)^{\mathbb{Z}_{+}}$to $\left(H, \phi^{*} \mu\right)^{\mathbb{Z}_{+}}$, such that the following diagram commutes,

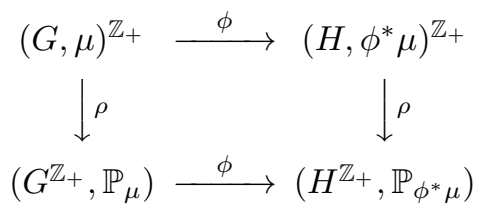

and so the lower map is also measure preserving. Therefore the images of sample paths in $H$ under $\phi$ are distributed according to a random walk on $H$ determined by the probability distribution $\phi^{*} \mu$.

An example of a random walk is the nearest neighbour random walk on a Cayley graph $\Gamma$ for $G$. This is the random walk determined by a probability distribution $\mu$ which gives equal weight to every generator and its inverse, and is zero on all the other elements of the group. In this case the support of the random walk is the whole group.

We now show that a sample path $\omega$ gives a sequence of points in $\widehat{G}$, which converges to a lamination $\lambda(\omega)$ in the Gromov boundary of the complex of curves, almost surely. Given a subset $X$ of $\mathcal{L}_{\text {min }}$, the measure of $X$ with respect to $\nu$ is defined to be the proportion of sample paths which converge to points contained in $X$. This measure $\nu$ is $\mu$-stationary, i.e., for any subset $X$ of $\mathcal{L}_{\text {min }}$,

$$
\nu(X)=\sum_{g \in G} \mu(g) \nu\left(g^{-1} X\right) .
$$

We will also refer to $\nu$ as a harmonic measure on $\mathcal{L}_{\text {min }}$.

Theorem 5.1. Consider a random walk on the mapping class group of a non-sporadic orientable surface of finite type, determined by a probability distribution $\mu$ such that the group generated by the support of $\mu$ is non-elementary. Then the sequence of points $\left\{w_{n}\right\}$ determined by a sample path $\omega$ converge to the boundary of the relative space $\widehat{G}$ with probability one, and the distribution of points on the boundary is given by a unique $\mu$-stationary measure $\nu$ on $\mathcal{L}_{\text {min }}$, the Gromov boundary of $\widehat{G}$.

Theorem 5.1 is an immediate consequence of results of Kaimanovich and Masur [KM96, and Klarreich [Kla], which we now describe. Kaimanovich and Masur consider the action of the mapping 
class group on Teichmüller space, and show that given a basepoint $x_{0} \in \mathcal{T}(\Sigma)$, then for almost all sample paths, the sequence of images of the basepoint $\left\{w_{n} x_{0}\right\}$ converges to a point in $\mathcal{P} \mathcal{M F}$, the Thurston compactification of Teichmüller space.

Theorem 5.2. KM96, Theorem 2.3.4] If $\mu$ is a probability measure on the mapping class group $G$ such that the group generated by its support is non-elementary, then there exists a unique $\mu$ stationary probability measure $\nu$ on the space $\mathcal{P} \mathcal{M F}$, which is purely non-atomic, and concentrated on the subset $\mathcal{U E} \subset \mathcal{P} \mathcal{M F}$ of uniquely ergodic foliations. For any $x \in \mathcal{T}(\Sigma)$ and almost every sample path $\omega=\left\{w_{n}\right\}$ of the random walk determined by $(G, \mu)$, the sequence $w_{n} x$ converges in $\mathcal{P} \mathcal{M F}$ to a limit $F(\omega) \in \mathcal{U} \mathcal{E}$, and the distribution of the limits $F(\omega)$ is given by $\nu$.

Kaimanovich and Masur [KM96] only state the result for closed surfaces, but Farb and Masur FM98 point out that the proof works for surfaces with punctures.

The following result of Klarreich Kla describes the Gromov boundary of the complex of curves, and will enable us to relate convergence in $\mathcal{P} \mathcal{M F}$ to convergence in the Gromov boundary of the complex of curves.

Theorem 5.3. Kla, Theorem 1.2 The inclusion map from $\mathcal{T}(\Sigma)$ to $\mathcal{T}_{\text {el }}(\Sigma)$ extends continuously to the portion $\mathcal{F}_{\text {min }}(\Sigma)$ of $\mathcal{P} \mathcal{M} \mathcal{F}(\Sigma)$ consisting of minimal foliations, to give a map $\pi: \mathcal{F}_{\text {min }}(\Sigma) \rightarrow$ $\partial \mathcal{T}_{\text {el }}(\Sigma)$. The map $\pi$ is surjective, and $\pi(F)=\pi(G)$ if and only if $F$ and $G$ are topologically equivalent. Moreover any sequence $\left\{x_{n}\right\}$ in $\mathcal{T}(\Sigma)$ that converges to a point in $\mathcal{P} \mathcal{M F}(\Sigma) \backslash \mathcal{F}_{\text {min }}(\Sigma)$ cannot accumulate in the electrified space onto any portion of $\partial \mathcal{T}_{\text {el }}(\Sigma)$.

A sample path $\omega$ gives rise to a sequence of points $\left\{w_{n} x_{0}\right\}$ in Teichmüller space which converge to a uniquely ergodic foliation $F(\omega)$, almost surely. Uniquely ergodic foliations are minimal, so the image of the sequence of points in $\mathcal{T}_{e l}$ converges to the same foliation $F(\omega)$. As $\mathcal{T}_{e l}$ is quasi-isometric to $\mathcal{C}(\Sigma)$, the sequence $\left\{w_{n} x_{0}\right\}$ in $\mathcal{T}_{e l}$ gives rise to a sequence in $\mathcal{C}(\Sigma)$, which lies a bounded distance from the images of $w_{n}$ in $\widehat{G}$. Therefore the sequence $w_{n}$ in $\widehat{G}$ converges almost surely to a point in the Gromov boundary corresponding to a uniquely ergodic foliation $F(\omega)$. This enables us to define a harmonic measure on the Gromov boundary $\mathcal{F}_{\text {min }}$, however, as the map from $\mathcal{P} \mathcal{M F}$ to $\mathcal{F}_{\text {min }}$ is a bijection when restricted to the uniquely ergodic foliations, this harmonic measure is the same as the pullback of $\nu$ under the map $\mathcal{P} \mathcal{M F} \rightarrow \mathcal{F}_{\text {min }}$. We will abuse notation and write $\nu$ for the harmonic measure on either $\mathcal{P} \mathcal{M F}$ or $\mathcal{F}_{\text {min }}$. As $\mathcal{P} \mathcal{M F}$ is essentially the same as $\mathcal{P} \mathcal{M L}$, and $\mathcal{F}_{\text {min }}$ is essentially the same as $\mathcal{L}_{\text {min }}$, we will also write $\nu$ for harmonic measure on these spaces as well.

The rest of this section is devoted to the proof of Theorem 1.1. In fact, we will prove the following more general result.

Theorem 5.4. Consider a random walk on the mapping class group of an orientable non-sporadic surface of finite type, determined by a probability distribution $\mu$, whose support generates a nonelementary subgroup. Let $R$ be a subset of $G$ with the property that every element of $R$ is conjugate to an element of relative length at most $B$, for some constant $B$. Then the probability that a random walk of length $n$ lies in $R$ tends to zero as $n$ tend to infinity.

The following observation shows that the set of periodic and reducible elements of the mapping class group form a set of elements which are conjugate to elements of bounded relative length, so Theorem 5.4 implies Theorem 1.1

Lemma 5.5. Every reducible or periodic element of the mapping class group of a surface which is not a sphere with three or fewer punctures is conjugate to an element of bounded relative length, where the bound only depends on the surface $\Sigma$. 
Proof. If $g$ is reducible, then $g$ preserves a collection of disjoint simple closed curves. We can conjugate $g$ so that one of these curves $y$ is distance 1 from the basepoint $x_{0}$ in $\mathcal{C}(\Sigma)$. As $g(y)$ is disjoint from $y$, and hence distance at most 1 from $y$, the image of the basepoint $x_{0}$ under $g$ is distance at most 3 from $x_{0}$, so $g$ is conjugate to an element of relative length at most $3 Q$, where $Q$ is the quasi-isometry constant between the relative metric and the complex of curves.

There are only finitely many conjugacy classes of periodic elements in the mapping class group of a given surface, so every periodic element is conjugate to a periodic element of bounded relative length, for some bound that depends on the surface.

We now prove Theorem 5.4. In Section 5.1 we show that the harmonic measure of the limit set of a centralizer is zero, assuming that there are infinitely many images of the limit set under the group generated by the support of the random walk. Finally in Section 5.2, we show that this implies that the asymptotic probability of the random walk being in $R$ tends to zero.

\subsection{Centralizers have harmonic measure zero}

In this section we show that if there are infinitely many images of the limit set of a centralizer $C(g)$ under the group generated by the support of the random walk, then $\overline{C(g)}$ has harmonic measure zero.

Lemma 5.6. Consider a random walk on the mapping class group $G$ of an orientable non-sporadic surface, determined by a probability distribution $\mu$, whose support generates a non-elementary subgroup $H$. Then if the limit set of a centralizer $\overline{C(g)}$ has infinitely many images under $H$, then $\nu(\overline{C(g)})=0$, where $\nu$ is the harmonic measure on $\mathcal{L}_{\text {min }}$ determined by $\mu$.

We do not assume that the probability distribution $\mu$ is symmetric, so the group $H$ generated by the support of $\mu$ may be larger than the semi-group generated by the support of $\mu$, which we shall denote by $H^{+}$. We shall write $H^{-}$for the semi-group generated by the inverses of elements in the support of $\mu$.

Proof. If $g$ is not periodic, then $\overline{C(g)}$ contains at most two points in the Gromov boundary $\mathcal{L}_{\text {min }}$, by Proposition 2.7, so $\overline{C(g)}$ has harmonic measure zero, as $\nu$ is non-atomic. We now consider the case in which $g$ is periodic, and it will then be convenient to consider arbitrary finite subgroups of the mapping class group, rather than just cyclic subgroups. We will argue that we may choose a finite subgroup $F$ such that the intersections of infinitely many distinct images of its limit sets have measure zero. The $\mu$-stationarity of the measure $\nu$ will then imply that all of these limit sets have $\nu$-measure zero.

Let $F$ be a maximal finite subgroup such that $\nu(\overline{C(F)})>0$. We now show that this implies that if any two distinct images of $\overline{C(F)}$ intersect, their intersection has measure zero with respect to $\nu$. The intersection of two images of $C(F)$ is the centralizer of the subgroup $F^{\prime}$ generated by the two conjugates of $F$, which is strictly larger than $F$ as the images are distinct. If the subgroup $F^{\prime}$ is finite, then it has a limit set of measure zero, as we have assumed that $F$ is a maximal finite subgroup with $\nu(\overline{C(F)})>0$. If the subgroup $F^{\prime}$ is infinite, then by Proposition 2.7 the limit set of the centralizer of $F^{\prime}$ consists of at most two points, and so has measure zero, as $\nu$ is non-atomic.

Let $s$ be the supremum of the harmonic measure of images of $\overline{C(F)}$ under the action of $H^{-}$, i.e. $s=\sup _{h \in H^{-}} \nu(h \overline{C(F)})$. The semi-group $H^{-}$generates $H$, so by Proposition 2.9, there are infinitely many distinct images of $\overline{C(F)}$ under the action of $H^{-}$, and furthermore, these images intersect in 
smaller centralizers, which have $\nu$-measure zero, by our assumption on the maximality of $F$. First suppose that the supremum is achieved. If $\nu(h \overline{C(F)})$ is equal to the supremum for some $h$, then as $\nu$ is $\mu$-stationary, this means that

$$
s=\nu(h \overline{C(F)})=\sum_{g \in H^{+}} \mu(g) \nu\left(g^{-1} h \overline{C(F)}\right) .
$$

We may take the sum over $H^{+}$as $\mu(g)=0$ for $g \notin H^{+}$. As $g^{-1}$ is in $H^{-}$, this implies that $\nu\left(g^{-1} h \overline{C(F)}\right) \leqslant s$, but as $\mu$ is a probability distribution with total mass one, if any $\nu\left(g^{-1} h \overline{C(F)}\right)$ is strictly less than $s$ for $\mu(g) \neq 0$, then the right hand sum is strictly less than $s$, a contradiction. The harmonic measure $\nu$ is $\mu^{(n)}$-stationary, for all $n$, so this implies that $\nu(g h \overline{C(F)})=s$ for all elements $g$ in the semi-group $H^{-}$, which contradicts the fact that $\nu$ has finite total mass. If the supremum is not achieved, then let $h_{i}$ be a sequence of elements in $H^{-}$such that $\nu\left(h_{i} \overline{C(F)}\right)$ tends to the supremum $s$, and we may assume we have chosen a sequence in which the $h_{i} \overline{C(F)}$ are all distinct. This gives infinitely many distinct sets each with measure bounded away from zero, and pairwise intersections of measure zero, which contradicts the fact that $\nu$ is a measure with finite total mass.

This completes the proof of Lemma 5.6 .

\subsection{Asymptotic probabilities}

A random walk is recurrent on a subset $X$ of $G$, if a sample path hits $X$ infinitely often with probability one. We say a random walk is transient on a subset $X$ of $G$, if a sample path hits $X$ infinitely often with probability zero.

We now observe that if the harmonic measure of the limit set of a set $X$ is zero, then the set $X$ is transient. In particular the probability that a random walk of length $n$ is in $X$ tends to zero as $n$ tend to infinity.

Lemma 5.7. Let $X$ be a subset of the mapping class group of a non-sporadic surface. If the harmonic measure of the closure of $X$ is zero, then the random walk is transient on $X$.

Proof. If a sample path converges to a lamination, and is also recurrent on $X$, i.e. hits $X$ infinitely often, then the limiting lamination must lie in the closure $\bar{X}$. As sample paths converge with probability one, the probability that a sample path is recurrent on $X$ is bounded above by the harmonic measure of $\bar{X}$. In particular. if the harmonic measure of $\bar{X}$ is zero, then the random walk is transient on $X$, i.e. a sample path hits $X$ finitely many times with probability one.

A sample path which never hits $X$ may still converge to a lamination lying in $\bar{X}$, so if the harmonic measure of $\bar{X}$ is greater than zero, this does not imply that sample paths are recurrent on $X$ with probability greater than zero.

We now prove Theorem 5.4, under the following assumption.

(*) The limit set of the centralizer of every non-trivial element of $G$ has infinitely many images under the group $H$ generated by the support of the random walk.

Recall that $R_{k}$ is the set of $k$-dense elements of $R$, so any two points in $R \backslash R_{k}$ are distance at least $k$ apart. We shall say $R \backslash R_{k}$ is a $k$-separated set. Furthermore, we have shown that the limit set of $R_{k}$ is contained in the limit set of a finite union of centralizers. As we have assumed that every $\overline{C(g)}$ has infinitely many images under $H$, Lemma 5.6 implies that each $\overline{C(g)}$ has harmonic 
measure zero, and so the limit set of $R_{k}$ also has harmonic measure zero. We want to show that the probability that you lie in $R$ in a low density region is small. The basic idea is that outside of $R_{k}$, the distance between elements of $R$ is at least $k$, so for a sample path outside $R_{k}$ there is some upper bound, depending on $k$, for how often the sample path hits elements of $R$, and furthermore, this upper bound tends to zero as $k$ tends to infinity.

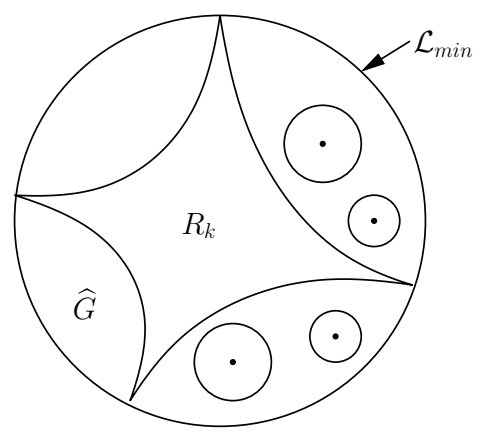

Figure 7: $R_{k}$ has harmonic measure zero.

Lemma 5.8. Let $X$ be a k-separated set in $G$. Then

$$
\mu^{(n)}(X) \leqslant \max \left\{\mu^{(m)}(g) \mid g \in B_{k / 2}\right\}+\mu^{(m)}\left(G \backslash B_{k / 2}\right)
$$

for all $m<n$, where $B_{k / 2}$ is the ball of radius $k / 2$ about the origin in the word metric.

Proof. The measure $\mu^{(n)}$ is the convolution of $\mu^{(n-m)}$ and $\mu^{(m)}$, i.e.

$$
\mu^{(n)}(X)=\sum_{g \in G} \mu^{(n-m)}(g) \mu^{(m)}\left(g^{-1} X\right),
$$

for any $m<n$. Any translate $g X$ of $X$ is also $k$-separated, so at most one point of $g X$ intersects the ball of radius $k / 2$ about the origin, therefore

$$
\mu^{(m)}(g X) \leqslant \max \left\{\mu^{(m)}(g) \mid g \in B_{k / 2}\right\}+\mu^{(m)}\left(G \backslash B_{k / 2}\right)
$$

for all $m$, and any $g \in G$. This implies $\mu^{(n)}(X) \leqslant \max \left\{\mu^{(m)}(g) \mid g \in B_{k / 2}\right\}+\mu^{(m)}\left(G \backslash B_{k / 2}\right)$, as required.

Let $s_{m}=\sup \left\{\mu^{(m)}(g) \mid g \in G\right\}$, which is an upper bound for $\max \left\{\mu^{(m)}(g) \mid g \in B_{k / 2}\right\}$. We now show that $s_{m}$ tends to zero as $m$ tends to infinity. If $s_{m}$ does not tend to zero, then there is a sequence $g_{m}$ with $\mu^{(m)}\left(g_{m}\right) \geqslant \epsilon>0$. Therefore there is a set of sample paths of positive measure (in fact of measure at least $\epsilon$ ) which are recurrent on any infinite subset of $\left\{g_{m}\right\}$. Choose a basepoint $x_{0}$ in Teichmüller space $\mathcal{T}(\Sigma)$, and consider the sequence of images $g_{m}\left(x_{0}\right)$. As sample paths converge to the boundary almost surely, the random walk is transient on bounded sets, so the distance in the Teichmüller metric between $x_{0}$ and $g_{m}\left(x_{0}\right)$ tends to infinity. Therefore we may pass to a subsequence such that $g_{m}\left(x_{0}\right)$ converges to a foliation $F$ in $\mathcal{P} \mathcal{M L}$. As $\nu$ is the weak-* limit of $\mu^{(n)}$ on $\mathcal{T}(\Sigma) \cup \mathcal{P} \mathcal{M L}$, this implies that the harmonic measure of $F$ is strictly larger than zero, 
contradicting the fact that the harmonic measure $\nu$ is non-atomic. This shows that $s_{m}$ tends to zero as $m$ tends to infinity.

To summarize, we have shown

$$
\begin{aligned}
\mathbb{P}\left(w_{n} \in R\right) & =\mathbb{P}\left(w_{n} \in R_{k}\right)+\mathbb{P}\left(w_{n} \in R \backslash R_{k}\right) \\
& \leqslant \mu^{(n)}\left(R_{k}\right)+s_{m}+\mu^{(m)}\left(G \backslash B_{k / 2}\right)
\end{aligned}
$$

for all $k$, and any $m<n$. The limit sets of centralizers have measure zero, so the harmonic measure of $R_{k}$ is zero, so $\mu^{(n)}\left(R_{k}\right)$ tends to zero as $n$ tends to infinity, for every $k$ and $m$. Therefore

$$
\lim _{n \rightarrow \infty} \mathbb{P}\left(w_{n} \in R\right) \leqslant s_{m}+\mu^{(m)}\left(G \backslash B_{k / 2}\right)
$$

for all $k$ and $m$. We have shown that $s_{m}$ tends to zero as $m$ tends to infinity, and we can choose a sequence $k_{m}$, which also tends to infinity as $m$ tends to infinity, such that $\mu^{(m)}\left(G \backslash B_{k_{m} / 2}\right)$ tends to zero. This implies that the probability that $w_{n}$ lies in $R$ tends to zero as $n$ tends to infinity, as required. This completes the proof of Theorem 5.4 assuming property $(*)$ above.

Finally, we prove Theorem 5.4 when there are limit sets of centralizers with finitely many images under the subgroup generated by the support of the random walk. We start by showing that the surface $\Sigma$ covers a surface $\mathcal{O}$ such that there is a homomorphism $\phi$ from $H$ to $G_{\mathcal{O}}$ with finite kernel, such that the image of $H$ in $G_{\mathcal{O}}$ satisfies property $(*)$, i.e. every non-trivial finite subgroup $\overline{C(F)}$ has infinitely many images under $\phi(H)$. Suppose there is a finite subgroup $F_{1}$ in $G$ such that $\overline{C\left(F_{1}\right)}$ has only finitely many images under $H$. Then by Proposition 2.9, the subgroup $H$ is contained in the normalizer $N\left(F_{1}\right)$, and so there is a map $\phi_{1}$ from $H$ to $G_{\mathcal{O}_{1}}$ with finite kernel, where $\mathcal{O}_{1}$ is the quotient surface $\Sigma / F_{1}$. We may repeat this process, i.e. if there is a non-trivial finite subgroup $F_{2}$ in $G_{\mathcal{O}_{1}}$ such that $\overline{C\left(F_{2}\right)}$ has only finitely many images under $\phi_{1}(H)$, then $\phi_{1}(H)$ is contained in $N\left(F_{2}\right)$, and so we obtain a homomorphism $\phi_{2} \circ \phi_{1}$ from $H$ to $G_{\mathcal{O}_{2}}$ with finite kernel, where $\mathcal{O}_{2}=\mathcal{O}_{1} / F_{2}$. However, the area of the hyperbolic surface is divided by the degree of the covering at each stage, and so this process terminates after finitely many steps, as there is a lower bound on the area of a hyperbolic orbifold. This gives the desired homomorphism from $H$ to $G_{\mathcal{O}}$ with finite kernel, for some surface $\mathcal{O}$ covered by $\Sigma$.

The homomorphism $\phi$ has finite kernel, so $\phi(H)$ is non-elementary subgroup of $G_{\mathcal{O}}$. The relative metric on $G_{\mathcal{O}}$ is quasi-isometric to the complex of curves $\mathcal{C}(\mathcal{O})$, and the map on curve complexes from $\mathcal{C}(\mathcal{O})$ to $\mathcal{C}(\Sigma)$ induced by the covering is a quasi-isometric embedding, by Rafi and Schleimer [RS09, so the relative metric on $H$ is also quasi-isometric to the relative metric on $\phi(H)$. Therefore the image of $\phi(R \cap H)$ is contained in a set $R^{\prime} \subset G_{\mathcal{O}}$ of elements which are conjugate to elements of bounded relative length, possibly for a different bound on the relative length. The homomorphism $\phi$ gives rise to an induced random walk on $G_{\mathcal{O}}$ determined by the probability distribution $\phi^{*} \mu(h)=\mu\left(\phi^{-1}(h)\right)$. Therefore, as the induced random walk on $G_{\mathcal{O}}$ satisfies property $(*)$, the probability that the image of the random walk lies in $R^{\prime}$ tends to zero, and so the probability that the original random walk lies in $R$ also tends to zero. This completes the proof of Theorem 5.4

\section{References}

[AAS07] James W. Anderson, Javier Aramayona, and Kenneth J. Shackleton, An obstruction to the strong relative hyperbolicity of a group, J. Group Theory 10 (2007), no. 6, 749-756. 
[BDM09] Jason Behrstock, Cornelia Druţu, and Lee Mosher, Thick metric spaces, relative hyperbolicity, and quasiisometric rigidity, Math. Ann. 344 (2009), no. 3, 543-595.

[Bir74] Joan S. Birman, Braids, links, and mapping class groups, Princeton University Press, Princeton, N.J., 1974. Annals of Mathematics Studies, No. 82.

[BH73] Joan S. Birman and Hugh M. Hilden, On isotopies of homeomorphisms of Riemann surfaces, Ann. of Math. (2) 97 (1973), 424-439.

[BLM83] Joan S. Birman, Alex Lubotzky, and John McCarthy, Abelian and solvable subgroups of the mapping class groups, Duke Math. J. 50 (1983), no. 4, 1107-1120.

[Bow] Brian H. Bowditch, Relatively hyperbolic groups.

[Bow05] Brian H. Bowditch, Hyperbolic 3-manifolds and the geometry of the curve complex, European Congress of Mathematics, Eur. Math. Soc., Zürich, 2005, pp. 103-115.

[Bow06] Brian H. Bowditch, Intersection numbers and the hyperbolicity of the curve complex, J. Reine Angew. Math. 598 (2006), 105-129.

[BH99] Martin R. Bridson and André Haefliger, Metric spaces of non-positive curvature, Grundlehren der Mathematischen Wissenschaften [Fundamental Principles of Mathematical Sciences], vol. 319, Springer-Verlag, Berlin, 1999.

[Deh38] M. Dehn, Die Gruppe der Abbildungsklassen, Acta Math. 69 (1938), no. 1, 135-206 (German). Das arithmetische Feld auf Flächen.

[EK74] Clifford J. Earle and Irwin Kra, On holomorphic mappings between Teichmüller spaces, Contributions to analysis (a collection of papers dedicated to Lipman Bers), Academic Press, New York, 1974, pp. 107-124.

[Far98] B. Farb, Relatively hyperbolic groups, Geom. Funct. Anal. 8 (1998), no. 5, 810-840.

[FM98] Benson Farb and Howard Masur, Superrigidity and mapping class groups, Topology 37 (1998), no. 6, 11691176.

[Fur71] Harry Furstenberg, Random walks and discrete subgroups of Lie groups, Advances in Probability and Related Topics, Vol. 1, Dekker, New York, 1971, pp. 1-63.

[Gro87] M. Gromov, Hyperbolic groups, Essays in group theory, Math. Sci. Res. Inst. Publ., vol. 8, Springer, New York, 1987, pp. 75-263.

[Ham06] Ursula Hamenstädt, Train tracks and the Gromov boundary of the complex of curves, Spaces of Kleinian groups, London Math. Soc. Lecture Note Ser., vol. 329, Cambridge Univ. Press, Cambridge, 2006, pp. 187207.

[Iva92] Nikolai V. Ivanov, Subgroups of Teichmüller modular groups, Translations of Mathematical Monographs, vol. 115, American Mathematical Society, Providence, RI, 1992. Translated from the Russian by E. J. F. Primrose and revised by the author.

[KM96] Vadim A. Kaimanovich and Howard Masur, The Poisson boundary of the mapping class group, Invent. Math. 125 (1996), no. 2, 221-264.

[KN04] Anders Karlsson and Guennadi A. Noskov, Some groups having only elementary actions on metric spaces with hyperbolic boundaries, Geom. Dedicata 104 (2004), 119-137.

[Ker83] Steven P. Kerckhoff, The Nielsen realization problem, Ann. of Math. (2) 117 (1983), no. 2, $235-265$.

[Kla] E. Klarreich, The boundary at infinity of the curve complex and the relative Teichmüller space.

[Kow08] E. Kowalski, The large sieve and its applications, Cambridge Tracts in Mathematics, vol. 175, Cambridge University Press, Cambridge, 2008. Arithmetic geometry, random walks and discrete groups.

[Lic64] W. B. R. Lickorish, A finite set of generators for the homeotopy group of a 2-manifold, Proc. Cambridge Philos. Soc. 60 (1964), 769-778.

[MH75] C. Maclachlan and W. J. Harvey, On mapping-class groups and Teichmüller spaces, Proc. London Math. Soc. (3) 30 (1975), no. part 4, 496-512.

[Mah10] Joseph Maher, Asymptotics for pseudo-Anosov elements in Teichmüller lattices, Geom. Funct. Anal. 20 (2010), no. 2, 527-544. 
[MM99] Howard A. Masur and Yair N. Minsky, Geometry of the complex of curves. I. Hyperbolicity, Invent. Math. 138 (1999), no. 1, 103-149.

[MM00] H. A. Masur and Y. N. Minsky, Geometry of the complex of curves. II. Hierarchical structure, Geom. Funct. Anal. 10 (2000), no. 4, 902-974.

[McC85] John McCarthy, A "Tits-alternative" for subgroups of surface mapping class groups, Trans. Amer. Math. Soc. 291 (1985), no. 2, 583-612.

[MP89] John McCarthy and Athanase Papadopoulos, Dynamics on Thurston's sphere of projective measured foliations, Comment. Math. Helv. 64 (1989), no. 1, 133-166.

[Osi06] Denis V. Osin, Relatively hyperbolic groups: intrinsic geometry, algebraic properties, and algorithmic problems, Mem. Amer. Math. Soc. 179 (2006), no. 843, vi+100.

[RS09] Kasra Rafi and Saul Schleimer, Covers and the curve complex, Geom. Topol. 13 (2009), no. 4, $2141-2162$.

[Riv08] Igor Rivin, Walks on groups, counting reducible matrices, polynomials, and surface and free group automorphisms, Duke Math. J. 142 (2008), no. 2, 353-379.

[Roy71] H. L. Royden, Automorphisms and isometries of Teichmüller space, Advances in the Theory of Riemann Surfaces (Proc. Conf., Stony Brook, N.Y., 1969), Ann. of Math. Studies, No. 66. Princeton Univ. Press, Princeton, N.J., 1971, pp. 369-383.

[Thu88] William P. Thurston, On the geometry and dynamics of diffeomorphisms of surfaces, Bull. Amer. Math. Soc. (N.S.) 19 (1988), no. 2, 417-431.

[Woe00] Wolfgang Woess, Random walks on infinite graphs and groups, Cambridge Tracts in Mathematics, vol. 138, Cambridge University Press, Cambridge, 2000.

[Zie73] Heiner Zieschang, On the homeotopy group of surfaces, Math. Ann. 206 (1973), 1-21. 